\title{
Domino Reactions That Combine an Azido-Schmidt Ring Expansion with the Diels-Alder Reaction
}

\author{
Yibin Zeng, D. Srinivasa Reddy, Erin Hirt, and Jeffrey Aubé* \\ Department of Medicinal Chemistry, 1251 Wescoe Hall Drive, Room 4070, Malott Hall, \\ University of Kansas, Lawrence, Kansas 66045-7582 \\ jaube@ku.edu
}

\section{Supporting Information}

Experimental details

Copies of ${ }^{1} \mathrm{H}$ and ${ }^{13} \mathrm{C}$ spectra of listed compounds

\section{Corresponding author:}

Professor Jeffrey Aubé

Department of Medicinal Chemistry

1251 Wescoe Hall Drive, Room 4070

Malott Hall

University of Kansas

Lawrence, Kansas 66045-2506

Tel 785.864.4496

Fax 785.864.5326

E-mail: jaube@ku.edu 


\section{Experimental Details}

General Information. The synthesis of the azido diene $\mathbf{1}^{1}$ and azido enone $\mathbf{1 9}^{2}$ have been described previously. Azido enones $\mathbf{1 0}$ and $\mathbf{1 1}$ were prepared via a HornerWadsworth-Emmons condensation of the known 3-azido propionaldehyde $\mathbf{A}^{3}$ and the corresponding $\beta$-keto phosphonates $\mathbf{B}^{4}$ and $\mathbf{C}^{5}$ Caution! All low molecular weight alkyl azides are potential explosion hazards and should be used with appropriate caution.

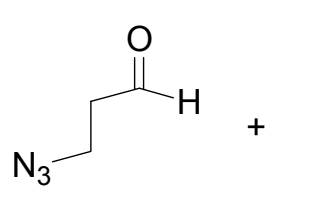

A

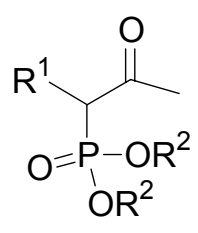

B, $\mathrm{R}^{1}=\mathrm{H}, \mathrm{R}^{2}=\mathrm{Me}$

C, $\mathrm{R}^{1}=\mathrm{Me}, \mathrm{R}^{2}=\mathrm{Et}$

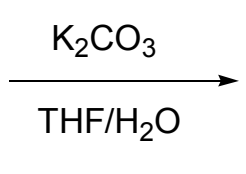

$10, \mathrm{R}=\mathrm{H}$

11, $R=M e$

(E)-6-Azido-3-hexen-2-one (10). To a solution of $\beta$-keto phosphonate $\mathbf{B}^{4}(1.60 \mathrm{~g}$, $10 \mathrm{mmol})$ in $\mathrm{THF} / \mathrm{H}_{2} \mathrm{O}(4: 1,40 \mathrm{~mL})$ was added $\mathrm{K}_{2} \mathrm{CO}_{3}(2.10 \mathrm{~g}, 15 \mathrm{mmol})$. The resulting solution was cooled in an ice bath followed by dropwise addition of the 3-azido propionaldehyde A $(1.0 \mathrm{~g}, 10 \mathrm{mmol})$ in THF $(4 \mathrm{~mL})$. The mixture was stirred in an ice bath for $1 \mathrm{~h}$ and then quenched with saturated aqueous $\mathrm{NaHCO}_{3}$. The reaction mixture was partitioned between water and EtOAc. The organic layer was dried $\left(\mathrm{Na}_{2} \mathrm{SO}_{4}\right)$, filtered, and concentrated to give an oil. Chromatography (10\% EtOAc/hexane) afforded $940 \mathrm{mg}(68 \%)$ of 10 as an oil. $\mathrm{R}_{f}=0.46\left(25 \%\right.$ EtOAc/hexane); ${ }^{1} \mathrm{H}$ NMR (400 MHz, $\left.\mathrm{CDCl}_{3}\right) \delta 2.25(\mathrm{~s}, 3 \mathrm{H}), 2.51(\mathrm{~m}, 2 \mathrm{H}), 3.44(\mathrm{t}, J=6.7 \mathrm{~Hz}, 2 \mathrm{H}), 6.15(\mathrm{~d}, J=16.0 \mathrm{~Hz}, 1 \mathrm{H})$, $6.74(\mathrm{~m}, 1 \mathrm{H}) ;{ }^{13} \mathrm{C} \mathrm{NMR}\left(100.6 \mathrm{MHz}, \mathrm{CDCl}_{3}\right) \delta 27.5,32.2,50.0,133.6,143.4,198.5$; IR (neat) $1709,1644 \mathrm{~cm}^{-1}$; MS (CI) $m / z 140\left(\mathrm{M}^{+}+1\right)$; HRMS calcd for $\mathrm{C}_{6} \mathrm{H}_{10} \mathrm{~N}_{3} \mathrm{O}\left(\mathrm{M}^{+}+\mathrm{H}\right)$ : 140.0824 , found 140.0853 . 
(E)-6-Azido-3-methyl-3-hexen-2-one (11). Azido enone 11 was prepared as described for 10 using $\beta$-keto phosphonate $\mathbf{C}^{5}$ (1.95 g, $\left.10 \mathrm{mmol}\right)$ to afford $310 \mathrm{mg}(20 \%)$ of 11 as an oil. $\mathrm{R}_{f}=0.47$ (25\% EtOAc/hexane); ${ }^{1} \mathrm{H}$ NMR (400 MHz, $\left.\mathrm{CDCl}_{3}\right) \delta 1.82(\mathrm{~s}$, $3 \mathrm{H}), 2.35(\mathrm{~s}, 3 \mathrm{H}), 2.57(\mathrm{~m}, 2 \mathrm{H}), 3.46(\mathrm{t}, J=6.7 \mathrm{~Hz}, 2 \mathrm{H}), 6.60(\mathrm{~m}, 1 \mathrm{H}) ;{ }^{13} \mathrm{C}$ NMR $(100.6$ $\left.\mathrm{MHz}, \mathrm{CDCl}_{3}\right) \delta 11.8,26.0,29.2,50.5,138.4,140.3,199.9$; IR (neat) 2097, $1668 \mathrm{~cm}^{-1}$; MS (CI) $m / z 154\left(\mathrm{M}^{+}+1\right)$; HRMS calcd for $\mathrm{C}_{7} \mathrm{H}_{12} \mathrm{~N}_{3} \mathrm{O}\left(\mathrm{M}^{+}+\mathrm{H}\right)$ : 154.0980, found 154.0966.

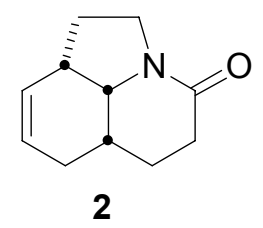

( \pm -1,2,5,6,6a,7,9a,9b-Octahydro-pyrrolo[3,2,1-i,j]quinolin-4-one (2). To a

solution of diene $\mathbf{1}^{1}$ (123 $\left.\mathrm{mg}, 1 \mathrm{mmol}\right)$ and 2-cyclopentenone (1 equiv) (200 mg, 1.4 mmol $)$ in $\mathrm{CH}_{2} \mathrm{Cl}_{2}(50 \mathrm{~mL})$ was added $\mathrm{MeAlCl}_{2}(1.0 \mathrm{M}$ in hexanes, $2.5 \mathrm{~mL})$ at $-78^{\circ} \mathrm{C}$. The resulting mixture was stirred at $-78^{\circ} \mathrm{C}$ for $2 \mathrm{~h}$ and then at room temperature for $12 \mathrm{~h}$. After quenching with aqueous $\mathrm{NaHCO}_{3}$, the mixture was partitioned between water and $\mathrm{CHCl}_{3}$. The organic layer was collected, dried $\left(\mathrm{Na}_{2} \mathrm{SO}_{4}\right)$, filtered and concentrated to give an oil. Chromatography (10-50\% EtOAc/hexane followed by $\left.1-2 \% \mathrm{MeOH} / \mathrm{CHCl}_{3}\right)$ afforded $68 \mathrm{mg}(38 \%)$ of 2 as an oil. $\mathrm{R}_{f}=0.45(1: 10 \mathrm{MeOH} / \mathrm{EtOAc}) ;{ }^{1} \mathrm{H}$ NMR (400 $\left.\mathrm{MHz}, \mathrm{CDCl}_{3}\right) \delta$ 1.70-2.05 (complex, 8H), $2.25(\mathrm{~m}, 1 \mathrm{H}), 2.42(\mathrm{~m}, 2 \mathrm{H}), 2.93(\mathrm{br}, \mathrm{s}, 1 \mathrm{H})$, $3.38(\mathrm{~m}, 1 \mathrm{H}), 3.56(\mathrm{~m}, 1 \mathrm{H}), 3.75(\mathrm{~m}, 1 \mathrm{H}), 5.43(\mathrm{dd}, J=1.2,10.1 \mathrm{~Hz}, 1 \mathrm{H}), 5.72(\mathrm{~m}, 1 \mathrm{H})$; ${ }^{13} \mathrm{C}$ NMR $\left(100.6 \mathrm{MHz}, \mathrm{CDCl}_{3}\right) \delta 23.0,26.1,27.7,28.5,28.9,39.4,44.2,59.4,127.1$, 127.4, 169.7; IR (neat) $1621 \mathrm{~cm}^{-1}$; MS (CI) m/z $178\left(\mathrm{M}^{+}+1\right)$; HRMS calcd for $\mathrm{C}_{11} \mathrm{H}_{16} \mathrm{NO}$ $\left(\mathrm{M}^{+}+\mathrm{H}\right): 178.1232$, found 178.1224 . 


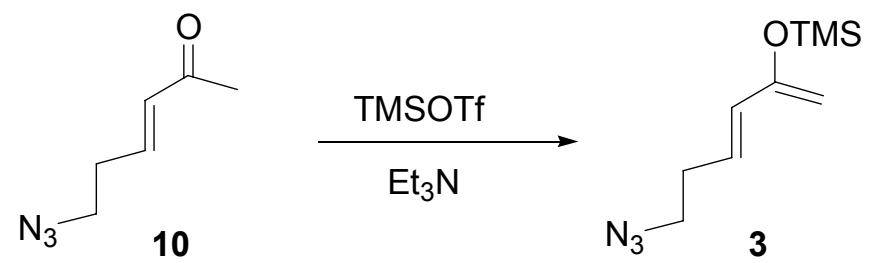

(E)-6-Azido-2-trimethylsilyloxy-1,3-hexadiene (3). To a solution of enone 10 $(1.4 \mathrm{~g}, 10 \mathrm{mmol})$ in anhydrous ethyl ether $(35 \mathrm{~mL})$ under argon was added $\mathrm{Et}_{3} \mathrm{~N}(2.1 \mathrm{~g}$, $20 \mathrm{mmol})$ at $0{ }^{\circ} \mathrm{C}$ followed by the addition of TMSOTf $(3.3 \mathrm{~g}, 15 \mathrm{mmol})$. The resulting mixture was stirred at $0{ }^{\circ} \mathrm{C}$ for $30 \mathrm{~min}$. The organic layer was washed with brine, dried $\left(\mathrm{Na}_{2} \mathrm{SO}_{4}\right)$, and evaporated under reduced pressure to give $2.18 \mathrm{~g}(98 \%)$ of $\mathbf{3}$ as an oil. This diene was used directly for the next step. $\mathrm{R}_{f}=0.52$ (2:98 EtOAc/hexane); ${ }^{1} \mathrm{H}$ NMR $\left(400 \mathrm{MHz}, \mathrm{CDCl}_{3}\right) \delta 0.26(\mathrm{~s}, 9 \mathrm{H}), 2.42(\mathrm{~m}, 2 \mathrm{H}), 3.36(\mathrm{t}, J=7.0 \mathrm{~Hz}, 2 \mathrm{H}), 4.31(\mathrm{~d}, J=3.5$ $\mathrm{Hz}, 2 \mathrm{H}), 5.92(\mathrm{~m}, 1 \mathrm{H}), 6.01(\mathrm{~d}, J=15.2 \mathrm{~Hz}, 1 \mathrm{H}) ;{ }^{13} \mathrm{C} \mathrm{NMR}\left(100.6 \mathrm{MHz}, \mathrm{CDCl}_{3}\right) \delta-0.1$, 31.5, 50.7, 95.3, 126.4, 130.5, 154.3; IR (neat) $2098 \mathrm{~cm}^{-1}$; MS (CI) $\mathrm{m} / z 212\left(\mathrm{M}^{+}+1\right)$; HRMS calcd for $\mathrm{C}_{9} \mathrm{H}_{18} \mathrm{~N}_{3} \mathrm{OSi}\left(\mathrm{M}^{+}+\mathrm{H}\right): 212.1219$, found 212.1196.

\section{General procedure for the intermolecular Diels-Alder/intramolecular}

Schmidt reaction involving diene 3 . To a cooled $\left(-78^{\circ} \mathrm{C}\right)$ solution of enone $(1.0-1.5$ mmol) in $\mathrm{CH}_{2} \mathrm{Cl}_{2}(15-20 \mathrm{~mL})$ under argon was added $\mathrm{SnCl}_{4}$ (1 equiv) followed by the addition of diene (1.8 equiv). The resulting mixture was stirred at $-78^{\circ} \mathrm{C}$ for $2 \mathrm{~h}$. After slow addition of another portion of $\mathrm{SnCl}_{4}$ (1.5 equiv), the mixture was stirred at room temperature for $12 \mathrm{~h}$, and then quenched with aqueous $\mathrm{NaHCO}_{3}$. The mixture was partitioned between water and $\mathrm{CHCl}_{3}$. The organic layer was collected, dried $\left(\mathrm{Na}_{2} \mathrm{SO}_{4}\right)$, filtered, and concentrated to give an oil. Chromatography (10-50\% EtOAc/hexane followed by $1-2 \% \mathrm{MeOH} / \mathrm{CHCl}_{3}$ ) afforded the desired lactams. 


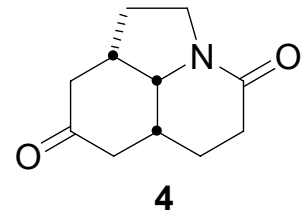

( \pm )-1,2,5,6,6a,7,9a,9b-Octahydro-pyrrolo[3,2,1-i,j]quinolin-4,8-dione (4). 2 -

Cyclopentenone (90 mg, $1.1 \mathrm{mmol})$ afforded $110 \mathrm{mg}(52 \%)$ of 4 as an oil. $\mathrm{R}_{f}=0.28(1: 10$ $\mathrm{MeOH} / \mathrm{EtOAc}$ ); ${ }^{1} \mathrm{H}$ NMR (400 MHz, $\left.\mathrm{CDCl}_{3}\right) \delta$ 1.70-1.82 (complex, $2 \mathrm{H}$ ), 2.01-2.18 (complex, 4H), 2.28-2.73 (complex, 5H), 3.62 (t, $J=10.2 \mathrm{~Hz}, 1 \mathrm{H}), 3.73$ (t, $J=4.8 \mathrm{~Hz}$, 1H), $3.80(\mathrm{~m}, 1 \mathrm{H}) ;{ }^{13} \mathrm{C} \mathrm{NMR}\left(100.6 \mathrm{MHz}, \mathrm{CDCl}_{3}\right) \delta 25.8,27.2,28.5,33.6,39.2,40.7$, 41.0, 43.1, 59.4, 169.0, 210.6; IR (neat) 1714, $1614 \mathrm{~cm}^{-1}$; MS (CI) $\mathrm{m} / z 194\left(\mathrm{M}^{+}+1\right)$; HRMS calcd for $\mathrm{C}_{11} \mathrm{H}_{16} \mathrm{NO}_{2}\left(\mathrm{M}^{+}+\mathrm{H}\right)$ : 194.1181 , found 194.1181.

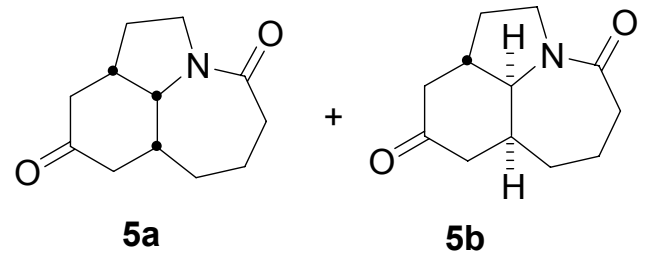

( \pm )-5-Octahydro-azipino[3,2,1-h,i]indol-4,9-dione (5). 2-Cyclohexenone (100 $\mathrm{mg}, 1.0 \mathrm{mmol})$ afforded $178 \mathrm{mg}(82 \%)$ of $\mathbf{5}$, an oil, as a ca. 3.4: $1(\mathbf{5 a}: \mathbf{5 b})$ mixture of diastereomers. The mixture solidified upon standing. $\mathrm{R}_{f}=0.32(1: 10 \mathrm{MeOH} / \mathrm{EtOAc}) ; \mathrm{IR}$ (neat) $1709,1624 \mathrm{~cm}^{-1}$; MS (CI) $\mathrm{m} / z 208\left(\mathrm{M}^{+}+1\right)$; HRMS calcd for $\mathrm{C}_{12} \mathrm{H}_{18} \mathrm{NO}_{2}\left(\mathrm{M}^{+}+\mathrm{H}\right)$ : 208.1338, found 208.1357. A pure sample of the major diastereomer was obtained after recrystallization from $\mathrm{CH}_{3} \mathrm{Cl} /$ EtOAc. $\mathrm{Mp} 131-133{ }^{\circ} \mathrm{C} ;{ }^{1} \mathrm{H}$ NMR $\left(500 \mathrm{MHz}, \mathrm{CDCl}_{3}\right) \delta$ 1.54-1.84 (complex, 6H), 2.00 (m, 1H), 2.11 (d, $J=15.7 \mathrm{~Hz}, 1 \mathrm{H}), 2.24$ (dd, $J=4.0,15.9$ $\mathrm{Hz}, 1 \mathrm{H}), 2.46(\mathrm{~m}, 3 \mathrm{H}), 2.65(\mathrm{~m}, 2 \mathrm{H}), 2.99(\mathrm{~m}, 1 \mathrm{H}), 3.24$ (two sets of $\mathrm{t}, J=6.56,11.9 \mathrm{~Hz}$, 1H), $3.91(\mathrm{~m}, 1 \mathrm{H}), 4.13(\mathrm{~d}, 1 \mathrm{H}, J=6.9 \mathrm{~Hz}) ;{ }^{13} \mathrm{C} \mathrm{NMR}\left(125.8 \mathrm{MHz}, \mathrm{CDCl}_{3}\right) \delta$ 18.5, 31.2, 33.6, 35.7, 38.0, 39.3, 39.7, 43.3, 45.8, 59.7, 174.3, 211.2. Minor diastereomer 
(diagnostic peaks only, from the mixture): ${ }^{1} \mathrm{H}$ NMR (400 MHz, $\left.\mathrm{CDCl}_{3}\right) \delta 3.51(\mathrm{~m}), 3.75$ (m); ${ }^{13} \mathrm{C}$ NMR (100.6 MHz, $\left.\mathrm{CDCl}_{3}\right) \delta$ 22.1, 26.6, 28.4, 31.9, 39.7, 43.2, 45.6, 47.3, 62.6, 171.6, 211.5.

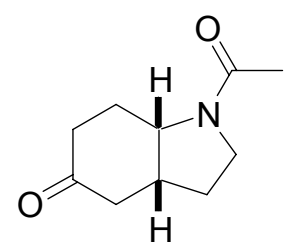

6

(士)-1-Acetyl-cis(3a, 7a)-octahydro-indol-5-one (6). Methyl vinyl ketone (100 $\mathrm{mg}, 1.43 \mathrm{mmol})$ afforded $155 \mathrm{mg}(60 \%)$ of $\mathbf{6}$, an oil, as a ca. 2.6:1 mixture of rotamers. $\mathrm{R}_{f}$ $=0.30(1: 10 \mathrm{MeOH} / \mathrm{EtOAc}) ; \mathrm{IR}$ (neat) 1708, $1623 \mathrm{~cm}^{-1}$; MS (CI) $\mathrm{m} / z 182\left(\mathrm{M}^{+}+1\right)$; HRMS calcd for $\mathrm{C}_{10} \mathrm{H}_{16} \mathrm{NO}_{2}\left(\mathrm{M}^{+}+\mathrm{H}\right)$ : 182.1181 , found 182.1199. Major rotamer: ${ }^{1} \mathrm{H}$ NMR (500 MHz, $\left.\mathrm{CDCl}_{3}\right) \delta 1.76(\mathrm{~m}, 3 \mathrm{H}), 2.06(\mathrm{~s}, 3 \mathrm{H}), 2.13(\mathrm{~m}, 1 \mathrm{H}), 2.30-2.51$ (complex, 4H), 2.61-2.80 (m, 2H), 3.46-3.60 (m, 2H), 4.37 (m, 1H); ${ }^{13} \mathrm{C}$ NMR (125.8 MHz, $\left.\mathrm{CDCl}_{3}\right)$ $\delta 22.5,25.5,30.7,36.9,37.0,41.6,46.6,54.7,169.4,210.9$. Minor rotamer (diagnostic peaks only): ${ }^{1} \mathrm{H}$ NMR $\left(500 \mathrm{MHz}, \mathrm{CDCl}_{3}\right) \delta 2.15(\mathrm{~s}), 2.25(\mathrm{~m}), 2.80(\mathrm{~m}), 4.12(\mathrm{~m}) ;{ }^{13} \mathrm{C}$ NMR $\left(125.8 \mathrm{MHz}, \mathrm{CDCl}_{3}\right) \delta 21.9,27.5,28.7,37.2,39.1,41.4,44.6,56.3,168.7,209.4$.

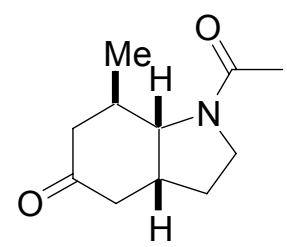

7

( \pm )-1-Acetyl-7-methyl-cis(3a,7a)-octahydro-indol-5-one (7). 3-Penten-2-one (100 mg, $1.2 \mathrm{mmol}$ ) afforded $137 \mathrm{mg}(59 \%)$ of 7 , an oil, as a ca. 2.4:1 mixture of rotamers. $\mathrm{R}_{f}=0.34$ (1:10 MeOH/EtOAc). IR (neat) 1712, $1627 \mathrm{~cm}^{-1}$; MS (CI) $\mathrm{m} / z 196$ $\left(\mathrm{M}^{+}+1\right)$; HRMS calcd for $\mathrm{C}_{11} \mathrm{H}_{18} \mathrm{NO}_{2}\left(\mathrm{M}^{+}+\mathrm{H}\right)$ : 196.1338, found 196.1338. Major 
rotamer: ${ }^{1} \mathrm{H}$ NMR $\left(500 \mathrm{MHz}, \mathrm{CDCl}_{3}\right) \delta 1.09(\mathrm{~d}, J=6.7 \mathrm{~Hz}, 3 \mathrm{H}), 1.76(\mathrm{~m}, 2 \mathrm{H}), 1.86(\mathrm{~m}$, 1H), $2.08(\mathrm{~s}, 3 \mathrm{H}), 2.05-2.41$ (complex, 3H), $2.68(\mathrm{~m}, 2 \mathrm{H}), 3.56(\mathrm{~m}, 2 \mathrm{H}), 4.30(\mathrm{~m}, 1 \mathrm{H})$; ${ }^{13} \mathrm{C}$ NMR $\left(125.8 \mathrm{MHz}, \mathrm{CDCl}_{3}\right) \delta 19.9,22.4,30.0,34.4,38.1,41.4,46.0,46.2,60.0$, 170.2, 210.0. Minor rotamer (diagnostic peaks only): ${ }^{1} \mathrm{H}$ NMR $\left(500 \mathrm{MHz}, \mathrm{CDCl}_{3}\right) \delta 1.12$ (d, $J=6.6 \mathrm{~Hz}), 2.17(\mathrm{~s}), 3.40(\mathrm{~m}), 3.79(\mathrm{~m}) ;{ }^{13} \mathrm{C} \mathrm{NMR}\left(125.8 \mathrm{MHz}, \mathrm{CDCl}_{3}\right) \delta$ 19.7, 22.5, $28.1,34.0,38.1,39.7,41.5,43.7,46.0,63.5$.

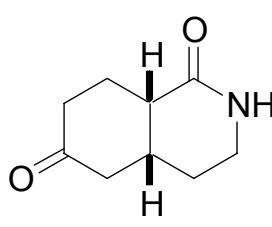

8

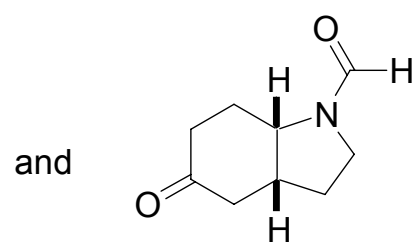

9

( \pm )-cis(4a, 8a)-Hexahydroisoquinolin-1,6-dione (8) and ( \pm )-1-Formyl-cis(3a,

7a)-octahydro-indol-5-one (9). Acrolein (90\%, $125 \mathrm{mg}, 2.0 \mathrm{mmol})$ was reacted using $\mathrm{MeAlCl}_{2}$ as Lewis acid in place of $\mathrm{SnCl}_{4}$ to afford $120 \mathrm{mg}(36 \%)$ of $\mathbf{8}$, as an oil. $\mathrm{R}_{f}=0.21$ (1:10 MeOH/EtOAc); ${ }^{1} \mathrm{H}$ NMR (400 MHz, $\left.\mathrm{CDCl}_{3}\right) \delta 1.76(\mathrm{~m}, 2 \mathrm{H}), 2.06$ (m, 2H), 2.342.58 (complex, 5H), $2.74(\mathrm{~m}, 1 \mathrm{H}), 3.42(\mathrm{~m}, 2 \mathrm{H}), 6.19\left(\mathrm{~s} \mathrm{br}, 1 \mathrm{H}, \mathrm{D}_{2} \mathrm{O}\right.$ exchangeable) ${ }^{13} \mathrm{C}$ NMR (100.6 MHz, $\left.\mathrm{CDCl}_{3}\right) \delta 25.9,26.8,35.3,39.5,40.2,41.0,44.1,173.3,210.9$; IR (neat) $1708,1649 \mathrm{~cm}^{-1}$; MS $\left(\mathrm{FAB}^{+}\right) \mathrm{m} / z 168\left(\mathrm{M}^{+}+1\right)$; HRMS calcd for $\mathrm{C}_{9} \mathrm{H}_{14} \mathrm{NO}_{2}$ $\left(\mathrm{M}^{+}+\mathrm{H}\right):$ 168.1025, found 168.1000 .

The same reaction also afforded $70 \mathrm{mg}(21 \%)$ of $\mathbf{9}$, an oil, as a ca. 1.3:1 mixture of rotamers. $\mathrm{R}_{f}=0.25(1: 10 \mathrm{MeOH} / \mathrm{EtOAc}) . \mathrm{IR}$ (neat) $1712,1658 \mathrm{~cm}^{-1}$; MS (FAB $\left.{ }^{+}\right) \mathrm{m} / \mathrm{z}$ $168\left(\mathrm{M}^{+}+1\right)$; HRMS calcd for $\mathrm{C}_{9} \mathrm{H}_{14} \mathrm{NO}_{2}\left(\mathrm{M}^{+}+\mathrm{H}\right)$ : 168.1025 , found 168.0983. Major rotamer: ${ }^{1} \mathrm{H}$ NMR (400 MHz, $\left.\mathrm{CDCl}_{3}\right) \delta 1.76(\mathrm{~m}, 2 \mathrm{H}), 2.13(\mathrm{~m}, 2 \mathrm{H}), 2.37$ (complex, 4H), 
$2.63(\mathrm{~m}, 2 \mathrm{H}), 3.60(\mathrm{~m}, 2 \mathrm{H}), 4.12(\mathrm{~m}, 1 \mathrm{H}), 8.28(\mathrm{~s}, 1 \mathrm{H}) ;{ }^{13} \mathrm{C} \mathrm{NMR}\left(100.6 \mathrm{MHz}, \mathrm{CDCl}_{3}\right) \delta$ 25.4, 28.9, 36.8, 38.5, 41.9, 43.0, 55.4, 161.0, 209.9. Minor rotamer (diagnostic peaks only): ${ }^{1} \mathrm{H}$ NMR (400 MHz, $\left.\mathrm{CDCl}_{3}\right) \delta 1.90(\mathrm{~m}), 2.21(\mathrm{~m}), 4.33(\mathrm{~m}), 8.35(\mathrm{~s}) ;{ }^{13} \mathrm{C} \mathrm{NMR}$ $\left(100.6 \mathrm{MHz}, \mathrm{CDCl}_{3}\right) \delta 29.5,30.8,36.6,37.0,42.1,45.4,53.6,161.5$.

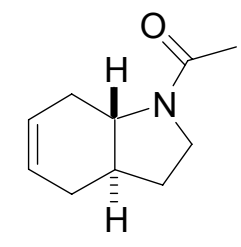

12

( \pm )-1-Acetyl-trans(3a, 7a)-2,3,3a,4,7,7a-hexahydro-1H-indole (12). Butadiene was bubbled through a solution of enone $\mathbf{1 0}(200 \mathrm{mg}, 1.4 \mathrm{mmol})$ in $\mathrm{CH}_{2} \mathrm{Cl}_{2}(50 \mathrm{~mL})$ at 0 ${ }^{\circ} \mathrm{C}$ for $5 \mathrm{~min}$ followed by dropwise addition of $\mathrm{MeAlCl}_{2}(1.0 \mathrm{M}$ in hexanes, $4.3 \mathrm{~mL})$. The resulting mixture was stirred at $0{ }^{\circ} \mathrm{C}$ for $2 \mathrm{~h}$ and then at room temperature for $26 \mathrm{~h}$. After dilution with $\mathrm{CH}_{2} \mathrm{Cl}_{2}(100 \mathrm{~mL})$, aqueous $\mathrm{NaHCO}_{3}$ was added. The organic layer was separated, washed with brine, and dried $\left(\mathrm{MgSO}_{4}\right)$. The organic layer was filtered, and concentrated to give $128 \mathrm{mg}(54 \%)$ of $\mathbf{1 2}$, a yellow oil, as a ca. $2.5: 1$ mixture of rotamers. $\mathrm{R}_{f}=0.5$ (EtOAc); IR (neat) 1418, 1443, $1633 \mathrm{~cm}^{-1}$; MS (FAB $\left.{ }^{+}\right) \mathrm{m} / z 166\left(\mathrm{M}^{+}+1\right)$; HRMS calcd. for $\mathrm{C}_{10} \mathrm{H}_{16} \mathrm{NO}\left(\mathrm{M}^{+}+\mathrm{H}\right)$ 166.1232, found 166.1221. Major rotamer: ${ }^{1} \mathrm{H}$ NMR (400 $\left.\mathrm{MHz}, \mathrm{CDCl}_{3}\right) \delta$ 1.46-1.49 (m, 2H), 1.89-1.95 (m, 1H), $1.98(\mathrm{~s}, 3 \mathrm{H}), 2.27-2.28(\mathrm{~m}, 2 \mathrm{H})$, 3.14-3.18 (m, 2H), $3.51(\mathrm{t}, J=9.4,1 \mathrm{H}), 5.60(\mathrm{~s}, 2 \mathrm{H}) ;{ }^{13} \mathrm{C} \mathrm{NMR}\left(100.6 \mathrm{MHz}, \mathrm{CDCl}_{3}\right) \delta$ 23.7, 30.5, 31.0, 33.0, 42.4, 48.9, 60.6, 126.5, 126.9, 170.7. Minor rotamer: ${ }^{1} \mathrm{H}$ NMR (400 MHz, $\mathrm{CDCl}_{3}$ ) (diagnostic peaks only) $\delta 2.01(\mathrm{~s}), 3.36-3.43(\mathrm{~m}), 3.83-3.88$ (dd, $J=$ 8.2 and $11.8 \mathrm{~Hz}) ;{ }^{13} \mathrm{C}$ NMR $\left(100.6 \mathrm{MHz}, \mathrm{CDCl}_{3}\right) \delta 23.4,29.2,34.2,44.1,47.8,125.3$, $127.9,170.8$. 


\section{General Procedure for the Diels-Alder/Schmidt reaction using enones 10, 11}

or 19. To a solution of azido enone $(\mathbf{1 0}, 11$ or $19 ; 0.4-2.1 \mathrm{mmol})$ and diene (3 equiv) in $\mathrm{CH}_{2} \mathrm{Cl}_{2}(15-20 \mathrm{~mL})$ at $0{ }^{\circ} \mathrm{C}$ was added $\mathrm{AlMeCl}_{2}$ (3 equiv). The resulting mixture was stirred at $0{ }^{\circ} \mathrm{C}$ for $2 \mathrm{~h}$ and then at room temperature for $12 \mathrm{~h}$, and then quenched with aqueous $\mathrm{NaHCO}_{3}$. The mixture was partitioned between water and $\mathrm{CHCl}_{3}$. The organic layer was collected, dried $\left(\mathrm{Na}_{2} \mathrm{SO}_{4}\right)$, filtered, and concentrated to give an oil. Chromatography $\left(10 \%\right.$ EtOAc/hexane followed by $\left.1-2 \% \mathrm{MeOH} / \mathrm{CHCl}_{3}\right)$ afforded the desired lactams.

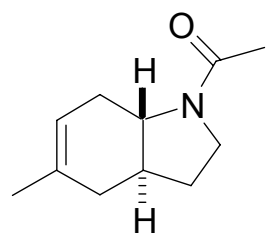

13

( \pm )-1-Acetyl-5-methyl-trans(3a,7a)-2,3,3a,4,7,7a-hexahydro-1H-indole (13).

Enone 10 (292 mg, $2.1 \mathrm{mmol})$ afforded $275 \mathrm{mg}(73 \%)$ of 13, a yellow oil, as a ca. 2.2:1 mixture of rotamers. $\mathrm{R}_{f}=0.25$ (EtOAc); IR (neat) $1417,1650 \mathrm{~cm}^{-1}$; MS (CI) $\mathrm{m} / z 180$ $\left(\mathrm{M}^{+}+1\right)$; HRMS calcd for $\mathrm{C}_{11} \mathrm{H}_{17} \mathrm{NO}\left(\mathrm{M}^{+}+\mathrm{H}\right)$ 180.1388, found 180.1385. Major rotamer: ${ }^{1} \mathrm{H}$ NMR $\left(400 \mathrm{MHz}, \mathrm{CDCl}_{3}\right) \delta 1.62(\mathrm{~s}, 3 \mathrm{H}), 1.73-1.95(\mathrm{~m}, 5 \mathrm{H}), 2.06-2.11(\mathrm{~m}, 2 \mathrm{H}), 3.42$ $(\mathrm{dt}, J=6.21,17.1 \mathrm{~Hz}, 2 \mathrm{H}), 3.52(\mathrm{t}, J=9.6 \mathrm{~Hz}, 1 \mathrm{H}), 5.29(\mathrm{~s}, 1 \mathrm{H}) ;{ }^{13} \mathrm{C} \mathrm{NMR}(100.6 \mathrm{MHz}$ $\left.\mathrm{CDCl}_{3}\right) \delta 23.6,23.7,30.4,32.5,35.7,43.0,49.2,60.8,121.0,133.9$, 170.7. Minor rotamer (diagnostic peaks only): ${ }^{1} \mathrm{H}$ NMR (400 $\left.\mathrm{MHz}, \mathrm{CDCl}_{3}\right) \delta 1.65$ (s), 2.00 (s), 3.24 $(\mathrm{dt}, J=5.7,17.7 \mathrm{~Hz}), 3.86(\mathrm{dd}, J=8.2,11.8 \mathrm{~Hz}) ;{ }^{13} \mathrm{C} \mathrm{NMR}\left(100.6 \mathrm{MHz}, \mathrm{CDCl}_{3}\right) \delta 23.3$, $23.6,29.2,33.8,35.8,44.6,48.1,60.6,119.4,135.5,170.8$. 


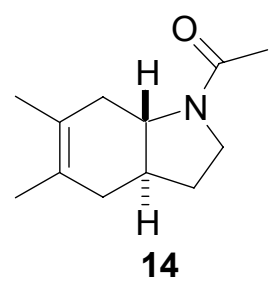

\section{( \pm -1-Acetyl-5,6-dimethyl-trans $(3 a, 7 a)-2,3,3 a, 4,7,7 a-h e x a h y d r o-1 H$-indole}

(14). Enone 10 (200 mg, $1.4 \mathrm{mmol})$ afforded $208 \mathrm{mg}(75 \%)$ of 14, a yellow oil, as a ca. 2.4:1 mixture of rotamers. $\mathrm{R}_{f}=0.28\left(\right.$ EtOAc); IR (neat) $1444,1650 \mathrm{~cm}^{-1} ; \mathrm{MS}\left(\mathrm{FAB}^{+}\right) \mathrm{m} / \mathrm{z}$ $194\left(\mathrm{M}^{+}+1\right)$; HRMS Calcd for $\mathrm{C}_{12} \mathrm{H}_{20} \mathrm{NO}\left(\mathrm{M}^{+}+\mathrm{H}\right)$ 194.1545, found 194.1551. Major rotamer: ${ }^{1} \mathrm{H}$ NMR (400 MHz, $\left.\mathrm{CDCl}_{3}\right) \delta 1.58(\mathrm{~s}, 6 \mathrm{H}), 1.85-1.97(\mathrm{~m}, 5 \mathrm{H}), 1.99(\mathrm{~s}, 3 \mathrm{H})$, 2.07-2.11 (m, 2H), $3.42(\mathrm{dt}, J=6.3,17.4 \mathrm{~Hz}, 1 \mathrm{H}), 3.52(\mathrm{t}, J=9.9 \mathrm{~Hz}, 2 \mathrm{H}) ;{ }^{13} \mathrm{C} \mathrm{NMR}$ $\left(100.6 \mathrm{MHz}, \mathrm{CDCl}_{3}\right) \delta 19.4,19.7,23.7,29.7,36.9,38.8,43.1,49.2,61.2,125.3,125.8$, 170.7. Minor rotamer (diagnostic peaks only): ${ }^{1} \mathrm{H}$ NMR (400 MHz, $\left.\mathrm{CDCl}_{3}\right) \delta 1.61(\mathrm{~s})$, $2.03(\mathrm{~s}) ;{ }^{13} \mathrm{C}$ NMR $\left(100.6 \mathrm{MHz}, \mathrm{CDCl}_{3}\right) \delta$ 19.9, 23.4, 29.2, 37.0, 40.1, 44.7, 48.1, 61.0, $124.2,126.9,170.8$.

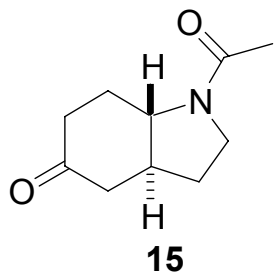

(士)-1-Acetyl-trans(3a, 7a)-octahydro-1H-indol-5-one (15). Enone 10 (275 mg, $2.0 \mathrm{mmol})$ afforded $220 \mathrm{mg}(71 \%)$ of $\mathbf{1 5}$, a yellow oil, as a ca. 1.4:1 mixture of rotamers. $\mathrm{R}_{f}=0.18$ (EtOAc); IR (neat) 1647, $1712 \mathrm{~cm}^{-1}$; MS (CI) $m / z 182\left(\mathrm{M}^{+}+\mathrm{H}\right)$; HRMS calcd for $\mathrm{C}_{10} \mathrm{H}_{16} \mathrm{NO}_{2}\left(\mathrm{M}^{+}+\mathrm{H}\right)$ 182.1181, found 182.1161. Major rotamer: ${ }^{1} \mathrm{H} \mathrm{NMR}(400 \mathrm{MHz}$, $\left.\mathrm{CDCl}_{3}\right) \delta 1.31-1.45(\mathrm{~m}, 2 \mathrm{H}), 1.91(\mathrm{~s}, 3 \mathrm{H}), 2.19-2.34(\mathrm{~m}, 3 \mathrm{H}), 2.46(\mathrm{~m}, 1 \mathrm{H}), 3.28-3.45(\mathrm{~m}$, 1H), 3.39-3.46 (m, 2H); ${ }^{13} \mathrm{C}$ NMR (100.6 MHz, $\left.\mathrm{CDCl}_{3}\right) \delta$ 23.6, 28.9, 30.2, 39.4, 45.3, 
45.5, 49.3, 62.3, 171.3, 209.4. Minor rotamer (diagnostic peaks only): ${ }^{1} \mathrm{H}$ NMR (400 $\left.\mathrm{MHz}, \mathrm{CDCl}_{3}\right) \delta 1.49-1.57(\mathrm{~m}), 1.89(\mathrm{~s}), 2.50(\mathrm{~m}), 3.00-3.06(\mathrm{~m}), 3.51-3.56(\mathrm{~m}) ;{ }^{13} \mathrm{C}$ NMR (400 MHz, $\left.\mathrm{CDCl}_{3}\right) \delta$ 23.3, 29.2, 30.4, 39.0, 45.2, 47.1, 48.83, 62.0, 170.9, 208.1.



16

( \pm )-1-Acetyl-7a-methyl-trans(3a, 7a)-octahydro-1H-indol-5-one (16). Enone $11(110 \mathrm{mg}, 0.7 \mathrm{mmol})$ afforded $85 \mathrm{mg}(61 \%)$ of $\mathbf{1 6}$ isolated as an oil. TLC (10:1 $\left.\mathrm{CHCl}_{3} / \mathrm{MeOH}\right): \mathrm{R}_{f}=0.50 .{ }^{1} \mathrm{H}$ NMR $\left(400 \mathrm{MHz}, \mathrm{CDCl}_{3}\right) \delta 1.33(\mathrm{~s}, 3 \mathrm{H}), 1.82(\mathrm{~m}, 2 \mathrm{H}), 1.92$ (m, 1H), $2.02(\mathrm{~s}, 3 \mathrm{H}), 2.16-2.53$ (complex, 5H), $3.13(\mathrm{~m}, 1 \mathrm{H}), 3.58(\mathrm{~m}, 2 \mathrm{H}) ;{ }^{13} \mathrm{C} \mathrm{NMR}$ $\left(100.6 \mathrm{MHz}, \mathrm{CDCl}_{3}\right) \delta 14.8,24.1,26.8,35.2,38.1,41.8,47.2,48.4,62.1,170.1,210.2$; IR (neat) $1709,1644 \mathrm{~cm}^{-1}$; MS (CI) $\mathrm{m} / z 196\left(\mathrm{M}^{+}+1\right)$; HRMS calcd for $\mathrm{C}_{11} \mathrm{H}_{18} \mathrm{NO}_{2}$ $\left(\mathrm{M}^{+}+\mathrm{H}\right):$ 196.1338, found 196.1332 .

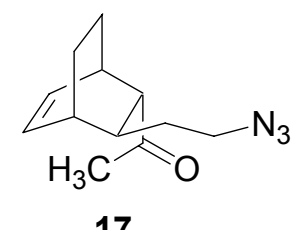

( \pm )-6-Acetyl-5-(2-azidoethyl)-bicyclo[2.2.2]oct-2-ene (17). Enone 10 (200 mg, $1.4 \mathrm{mmol})$ afforded $247 \mathrm{mg}(79 \%)$ of $\mathbf{1 7}$ as a dark yellow oil. $\mathrm{R}_{f}=0.64(1: 4 \mathrm{EtOAc} /$ hexanes); ${ }^{1} \mathrm{H}$ NMR (400 MHz, $\left.\mathrm{CDCl}_{3}\right) \delta$ 1.56-1.70 (m, 8H), 2.17 (s, 3H), 2.42-2.45 (m, $1 \mathrm{H}), 2.86-2.88(\mathrm{~m}, 1 \mathrm{H}), 3.20-3.33(\mathrm{~m}, 2 \mathrm{H}), 6.06(\mathrm{t}, J=7.0 \mathrm{~Hz}, 1 \mathrm{H}), 6.41(\mathrm{t}, J=7.1 \mathrm{~Hz}$, $1 \mathrm{H}) ;{ }^{13} \mathrm{C}$ NMR $\left(100.6 \mathrm{MHz}, \mathrm{CDCl}_{3}\right) \delta 18.9,26.6,28.8,33.6,33.9,34.1,36.1,50.7,59.6$, 
130.6, 137.2, 209.6; IR (neat) 1708, $2096 \mathrm{~cm}^{-1}$. MS (CI) $\mathrm{m} / z 220\left(\mathrm{M}^{+}+\mathrm{H}\right)$; HRMS calcd for $\mathrm{C}_{12} \mathrm{H}_{18} \mathrm{~N}_{3} \mathrm{O}\left(\mathrm{M}^{+}+\mathrm{H}\right)$ 220.1450, found 220.1453.

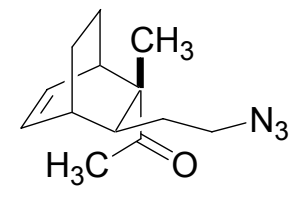

18

( \pm )-6-Methyl-6-acetyl-5-(2-azidoethyl)-bicyclo[2.2.2]oct-2-ene (18). Enone 11

(110 mg, $0.72 \mathrm{mmol})$ afforded $120 \mathrm{mg}(72 \%)$ of $\mathbf{1 8}$ as a dark yellow oil. $\mathrm{R}_{f}=0.67(1: 4$ EtOAc/hexanes); ${ }^{1} \mathrm{H}$ NMR (400 MHz, $\left.\mathrm{CDCl}_{3}\right) \delta 1.04-1.19$ (m, 2H), $1.14(\mathrm{~s}, 3 \mathrm{H}), 1.59$ $1.82(\mathrm{~m}, 5 \mathrm{H}), 2.13(\mathrm{~s}, 3 \mathrm{H}), 2.20(\mathrm{~m}, 1 \mathrm{H}), 2.40(\mathrm{~s} \mathrm{br}, 1 \mathrm{H}), 2.66(\mathrm{~s} \mathrm{br}, 1 \mathrm{H}), 3.33(\mathrm{~m}, 2 \mathrm{H})$, $6.25(\mathrm{~m}, 2 \mathrm{H}) ;{ }^{13} \mathrm{C} \mathrm{NMR}\left(100.6 \mathrm{MHz}, \mathrm{CDCl}_{3}\right) \delta 17.9,19.8,20.6,25.4,28.4,33.5,37.1$, 38.8, 51.1, 53.1, 133.7, 136.2, 212.9; IR (neat) 1702, $2094 \mathrm{~cm}^{-1}$. MS (CI) $\mathrm{m} / z 234\left(\mathrm{M}^{+}+\right.$ H); HRMS calcd for $\mathrm{C}_{13} \mathrm{H}_{20} \mathrm{~N}_{3} \mathrm{O}\left(\mathrm{M}^{+}+\mathrm{H}\right)$ 234.1606, found 234.1594.

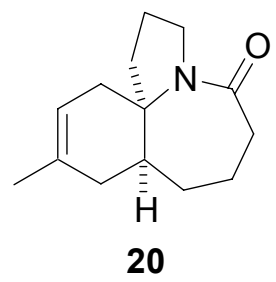

( \pm )-12-Methyl-5-aza-tricyclo[8.4.0.0 ${ }^{1,5} \mid$ tetradec-12-en-6-one (20). Enone $19^{2}$ $(180 \mathrm{mg}, 1.0 \mathrm{mmol})$ afforded $185 \mathrm{mg}(84 \%)$ of 20 as an oil. $\mathrm{R}_{f}=0.47(10: 1$ $\left.\mathrm{CHCl}_{3} / \mathrm{MeOH}\right) ;{ }^{1} \mathrm{H} \mathrm{NMR}\left(400 \mathrm{MHz}, \mathrm{CDCl}_{3}\right) \delta$ 1.51-1.79 (complex, $\left.12 \mathrm{H}\right), 1.96(\mathrm{~m}, 1 \mathrm{H})$, $2.16(\mathrm{~m}, 1 \mathrm{H}), 2.47(\mathrm{~m}, 3 \mathrm{H}), 2.61(\mathrm{~m}, 1 \mathrm{H}), 3.36(\mathrm{~m}, 1 \mathrm{H}), 3.90(\mathrm{~m}, 1 \mathrm{H}), 5.25(\mathrm{~m}, 1 \mathrm{H}) ;{ }^{13} \mathrm{C}$ NMR (100.6 MHz, $\left.\mathrm{CDCl}_{3}\right) \delta$ 20.7, 23.8 (two peaks), 32.0, 34.1, 39.2, 39.4, 40.8, 43.5, 
49.6, 64.6, 119.1, 132.4, 174.2; IR (neat) $1626 \mathrm{~cm}^{-1}$; MS (CI) $\mathrm{m} / z 220\left(\mathrm{M}^{+}+1\right)$; HRMS calcd for $\mathrm{C}_{14} \mathrm{H}_{22} \mathrm{NO}\left(\mathrm{M}^{+}+\mathrm{H}\right): 220.1701$, found 220.1701 .

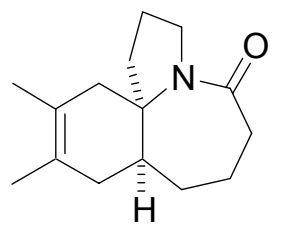

21

\section{( \pm )-12, 13-Dimethyl-5-aza-tricyclo[8.4.0.0 $\left.{ }^{1,5}\right]$ tetradec-12-en-6-one (21).}

Enone $19(180 \mathrm{mg}, 1.0 \mathrm{mmol})$ afforded $198 \mathrm{mg}(85 \%)$ of 21 as an oil. $\mathrm{R}_{f}=0.48(10: 1$ $\left.\mathrm{CHCl}_{3} / \mathrm{MeOH}\right) ;{ }^{1} \mathrm{H} \mathrm{NMR}\left(400 \mathrm{MHz}, \mathrm{CDCl}_{3}\right) \delta$ 1.51-1.90 (complex, $\left.16 \mathrm{H}\right), 2.12(\mathrm{~m}, 1 \mathrm{H})$, $2.47(\mathrm{~m}, 3 \mathrm{H}), 2.63(\mathrm{dd}, J=8.3,14.6 \mathrm{~Hz}, 1 \mathrm{H}), 3.38(\mathrm{~m}, 1 \mathrm{H}), 3.90(\mathrm{~m}, 1 \mathrm{H}) ;{ }^{13} \mathrm{C} \mathrm{NMR}$ $\left(100.6 \mathrm{MHz}, \mathrm{CDCl}_{3}\right) \delta 19.2,19.4,20.7,23.8,32.0,34.1,38.1,39.2,40.9,41.0,43.8$, 49.6, 65.1, 123.4, 123.8, 174.3; IR (neat) $1624 \mathrm{~cm}^{-1}$; MS (CI) $\mathrm{m} / z 234\left(\mathrm{M}^{+}+1\right)$; HRMS calcd for $\mathrm{C}_{15} \mathrm{H}_{24} \mathrm{NO}\left(\mathrm{M}^{+}+\mathrm{H}\right): 234.1858$, found 234.1850.

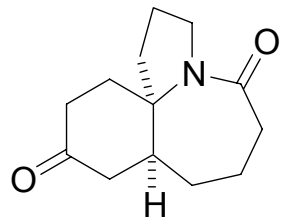

22

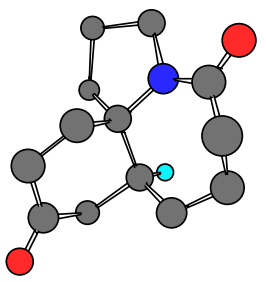

( \pm )-5-Aza-tricyclo[8.4.0.0 $\left.{ }^{1,5}\right]$ tetradecane-6,12-dione (22). Enone 19 (70 mg, 0.4 mmol) afforded $80 \mathrm{mg}(91 \%)$ of 22 as an oil which solidified upon standing. The structure 22 was determined by X-ray crystallography after recrystallization from MeOH/EtOAc. Mp 136-138 ${ }^{\circ} \mathrm{C} ; \mathrm{R}_{f}=0.41\left(10: 1 \mathrm{CHCl}_{3} / \mathrm{MeOH}\right) ;{ }^{1} \mathrm{H}$ NMR $(400 \mathrm{MHz}$, $\left.\mathrm{CDCl}_{3}\right) \delta 1.54-1.64(\mathrm{~m}, 2 \mathrm{H}), 1.80-2.06$ (complex, 7H), $2.25(\mathrm{~m}, 1 \mathrm{H}), 2.421-2.52(\mathrm{~m}, 4 \mathrm{H})$ $2.64(\mathrm{~m}, 2 \mathrm{H}), 2.76(\mathrm{dd}, J=5.1,13.2 \mathrm{~Hz}, 1 \mathrm{H}), 3.49(\mathrm{~m}, 1 \mathrm{H}), 3.93(\mathrm{~m}, 1 \mathrm{H}) ;{ }^{13} \mathrm{C} \mathrm{NMR}$ $\left(100.6 \mathrm{MHz}, \mathrm{CDCl}_{3}\right) \delta 20.9,22.3,28.4,33.9,38.4,38.6,40.3,45.8,48.2,49.6,64.9$, 
173.9, 209.2; IR (neat) 1705, $1613 \mathrm{~cm}^{-1}$; MS (CI) $\mathrm{m} / z 222\left(\mathrm{M}^{+}+1\right)$; HRMS calcd for $\mathrm{C}_{13} \mathrm{H}_{20} \mathrm{NO}_{2}\left(\mathrm{M}^{+}+\mathrm{H}\right): 222.1494$, found 222.1484.

\section{References}

(1) Grieco, P. A.; Galatsis, P.; Spohn, R. F. Tetrahedron 1986, 42, 2847-2853.

(2) Milligan,G. L.; Mossman, C. J.; Aubé, J. J. Am. Chem. Soc. 1995, 117, 10449-10459.

(3) Davies, A. J.; Donald, A. S. R.; Marks, R. E. J. Chem. Soc. Sect.C 1967, $2109-2112$.

(4) Corbel, B.; Medinger, L.; Haelters, J. P.; Sturtz, G. Synthesis 1985, 1048-1051.

(5) Clark, R. D.; Kozar, L. G.; Heathcock, C. H. Synthesis 1975, 635-636. 

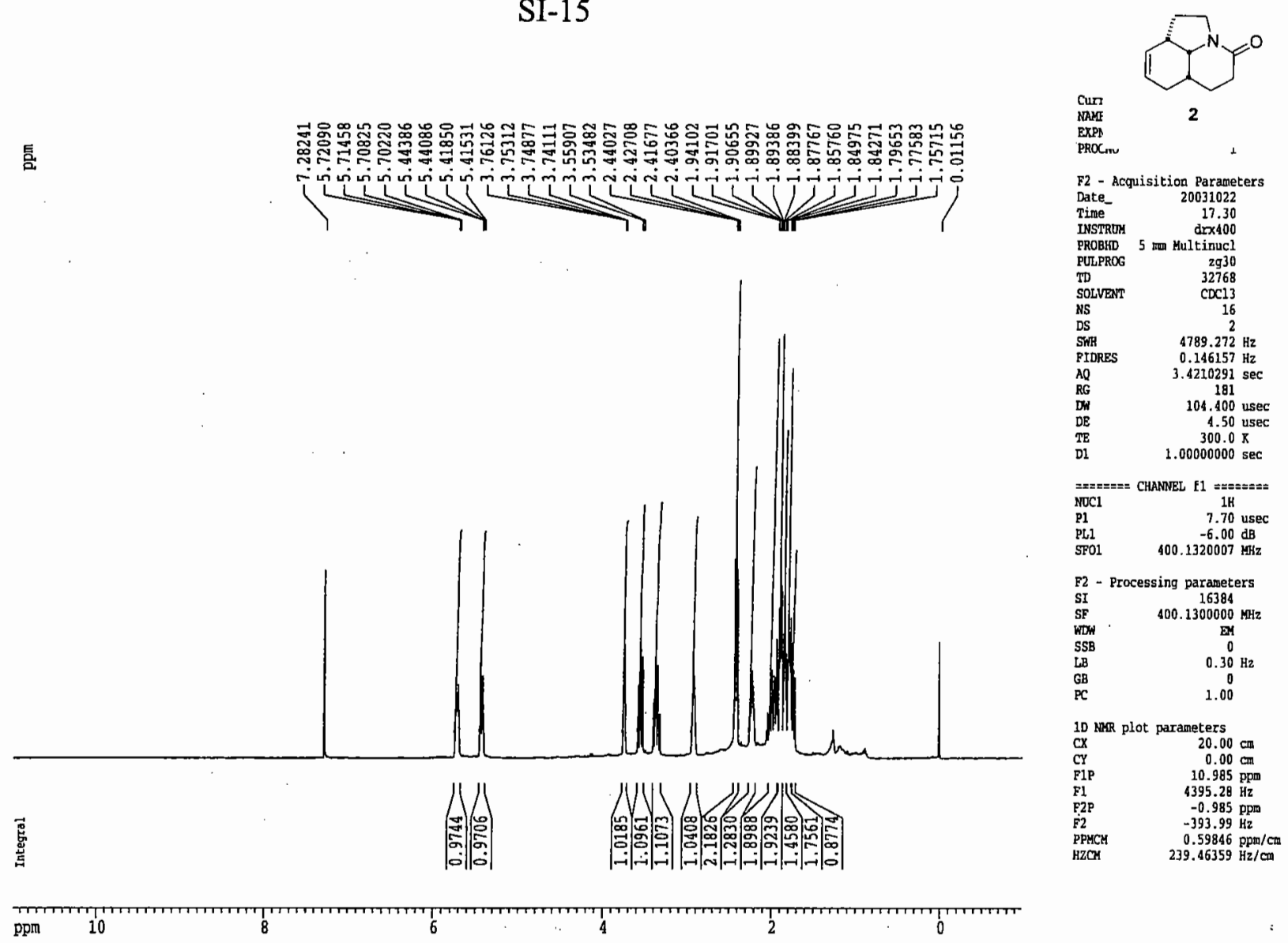

$0.05000000 \mathrm{sec}$ $0.00002000 \mathrm{sec}$
0.000

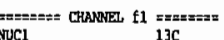


SI-16

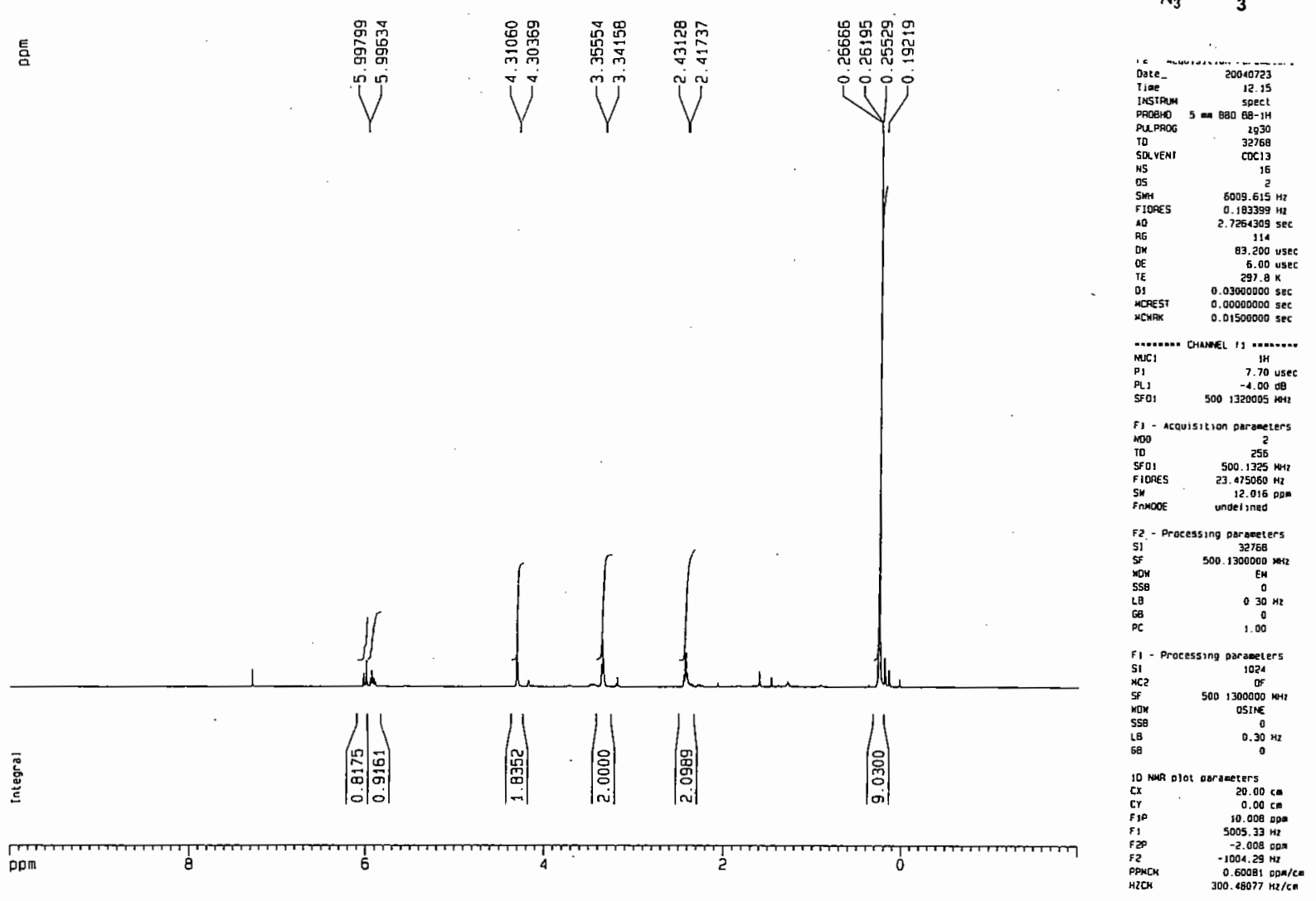

言

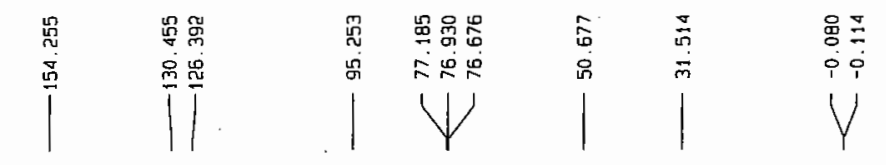
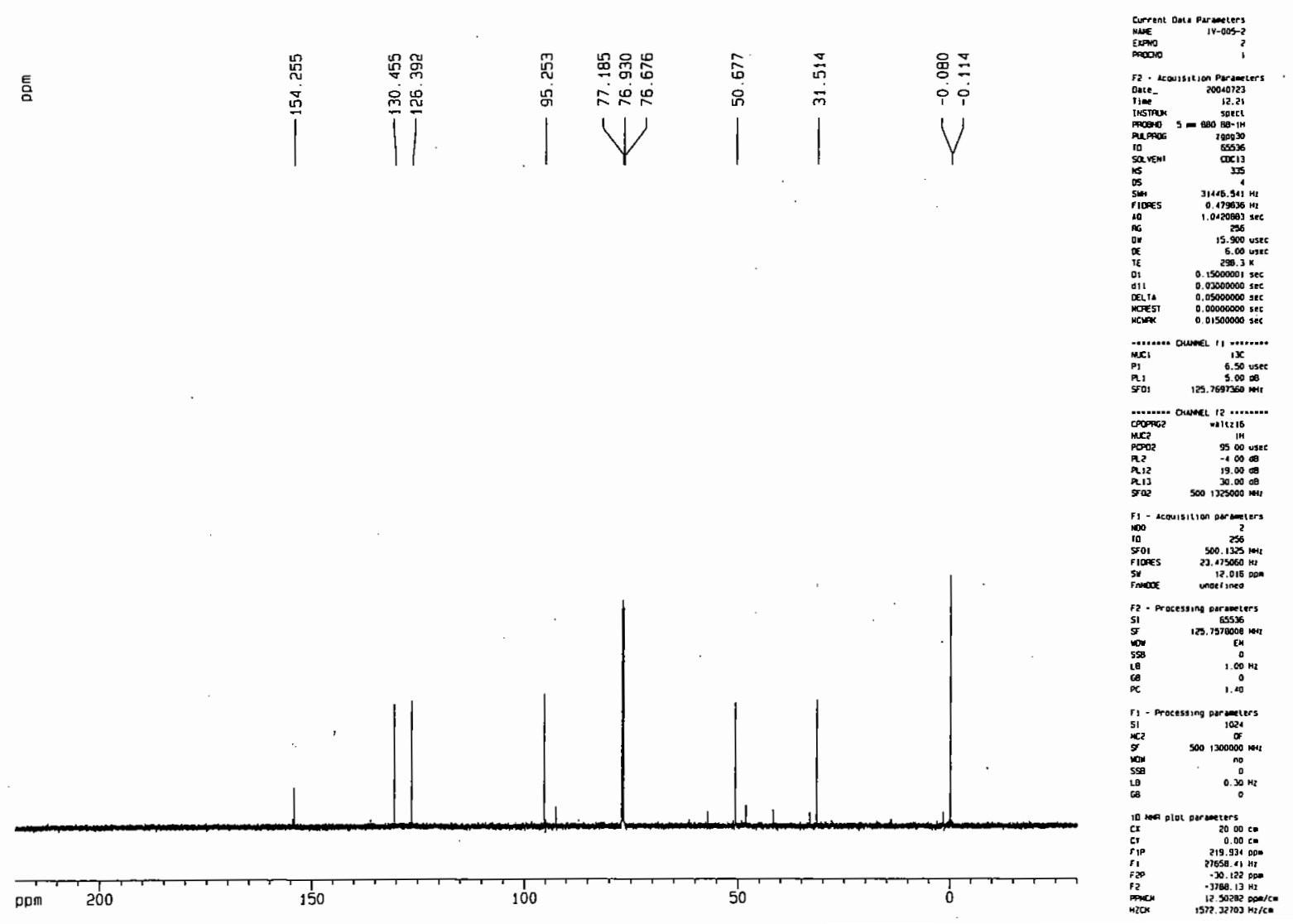
镸
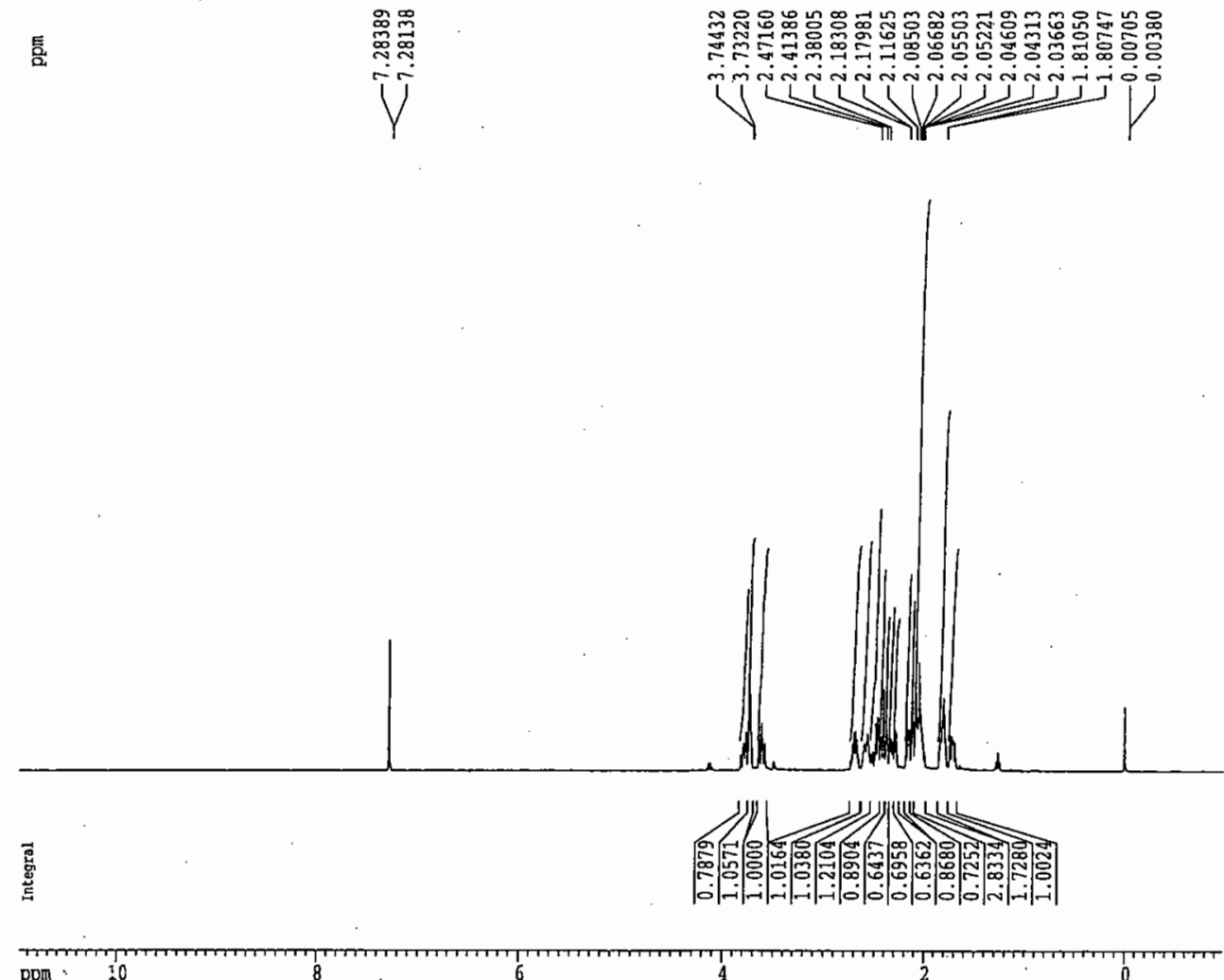


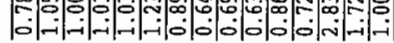

ppm : $\quad 10$

镸
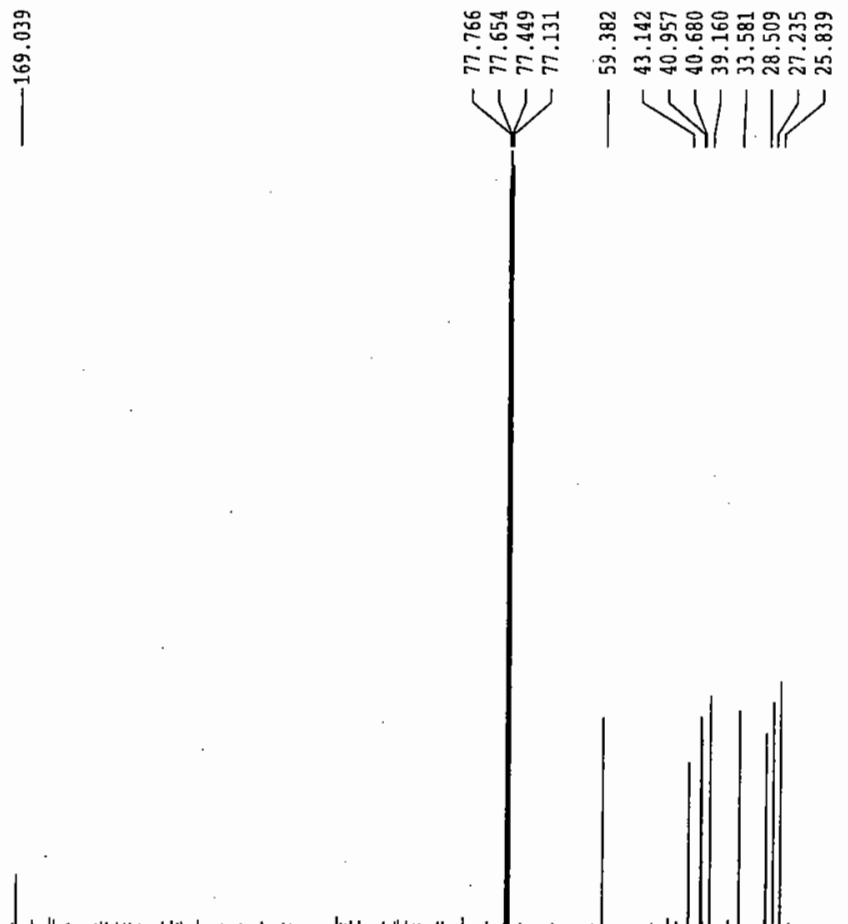

ppm 225

10

175

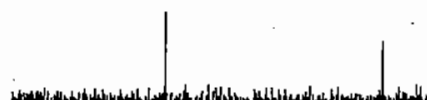

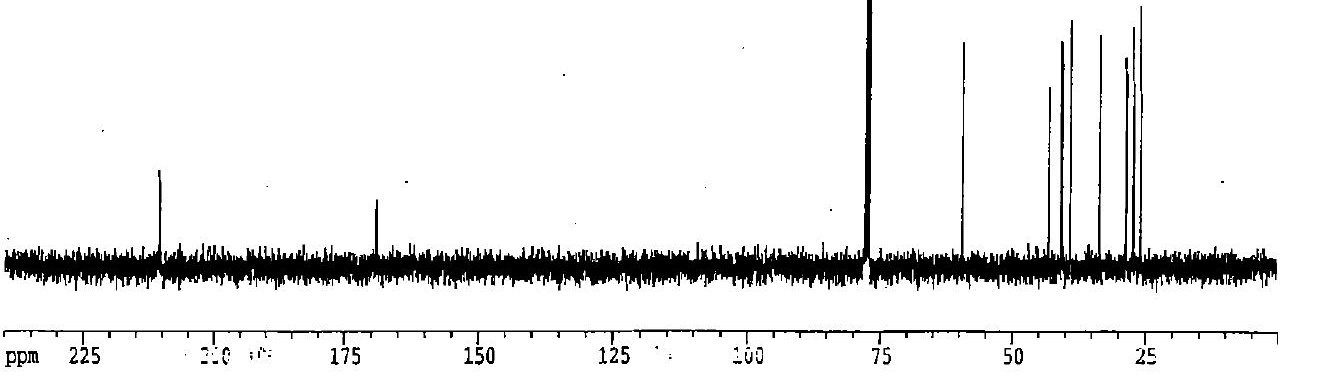

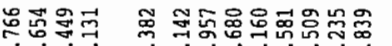

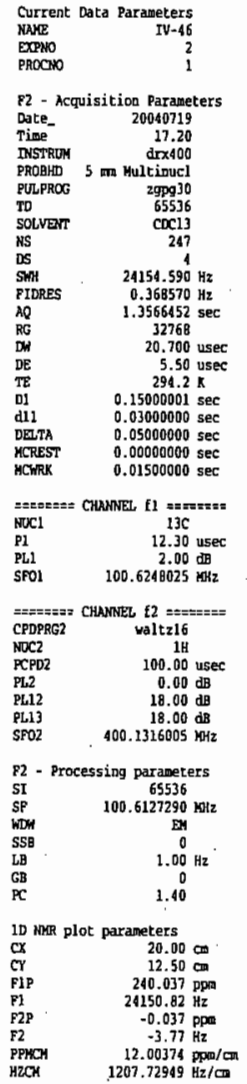




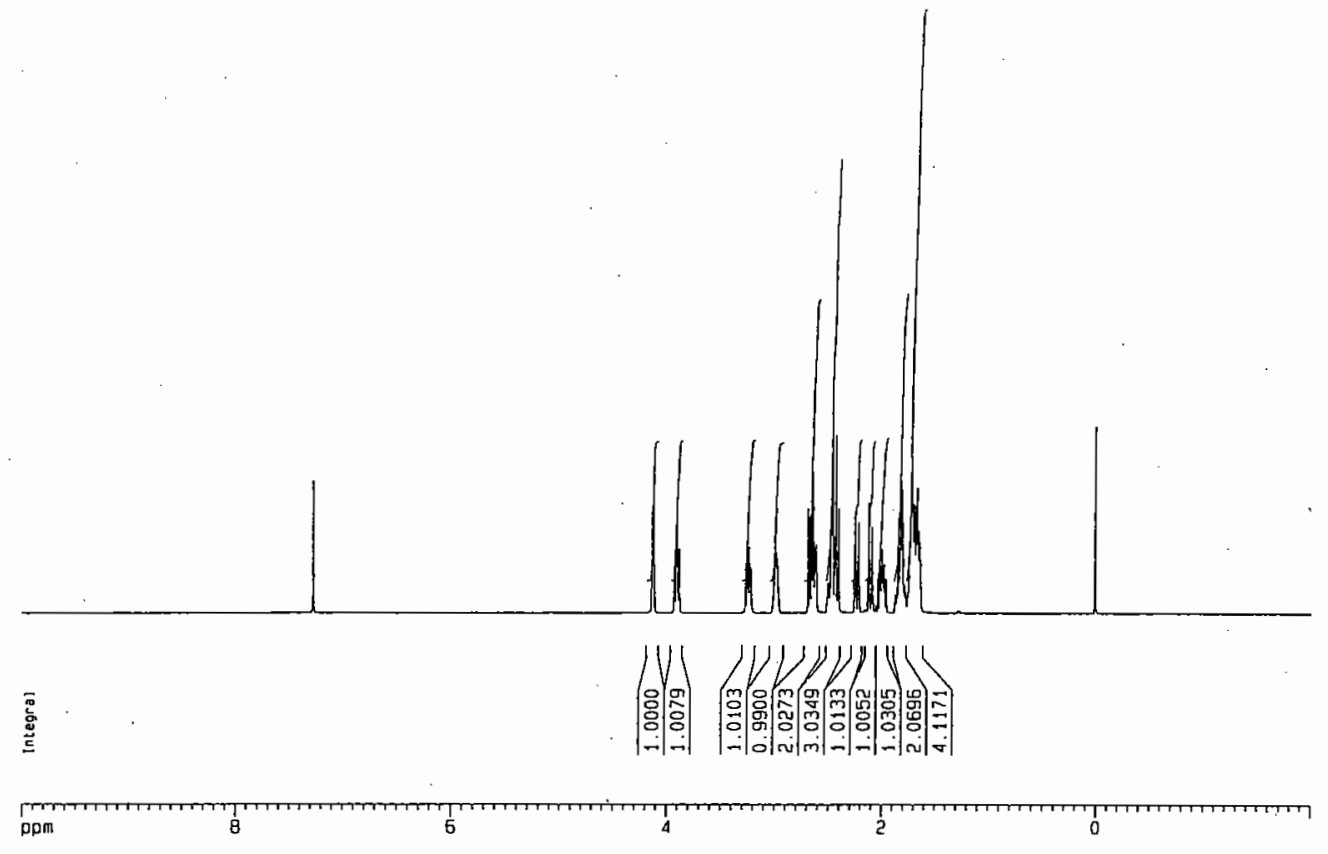

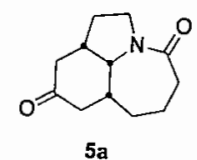

$5 \mathbf{a}$
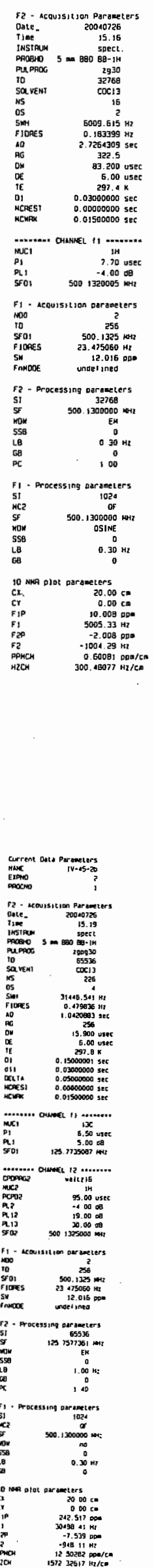


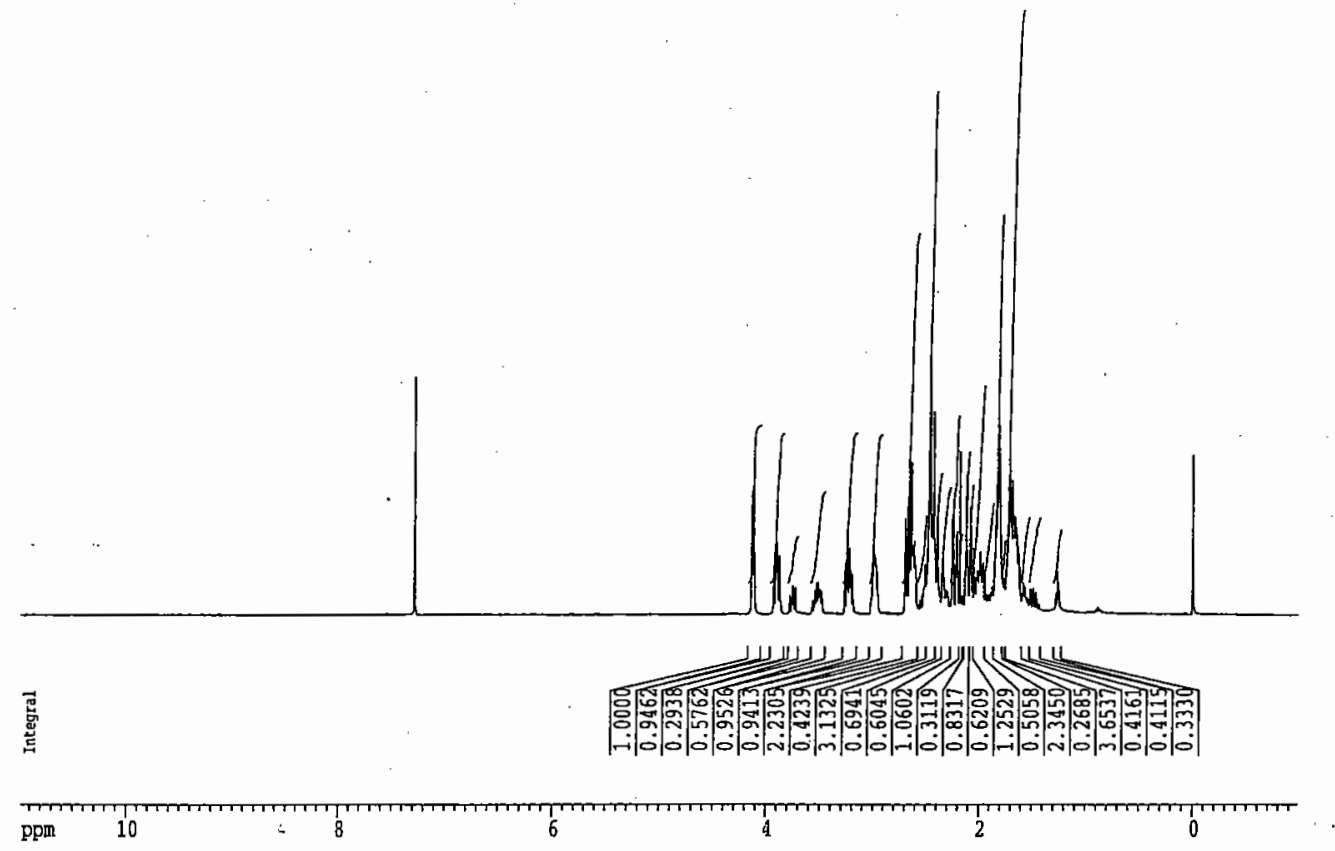

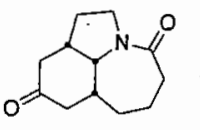

$5 \mathbf{a}$

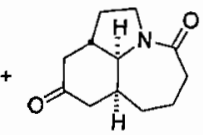

5b

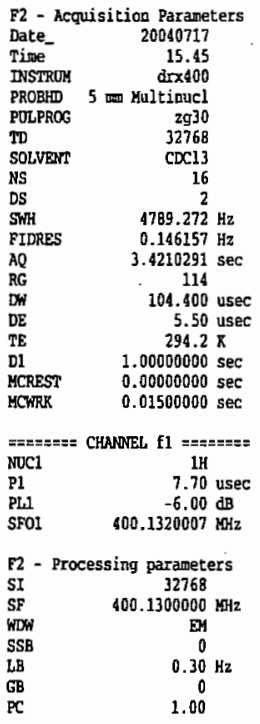

1D NRR plot parameters

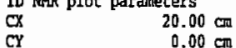

F1P $\quad 10.985 \mathrm{cpm}$

$\begin{array}{ll}\text { F2P } & 4395.28 \mathrm{~Hz} \\ \text { F2 } & -0.985 \mathrm{ppm} \\ \text { PegCr } & -393.99 \mathrm{~Hz}\end{array}$

PPMCY $\quad 0.59846 \mathrm{ppra} / \mathrm{cm}$

HzOS $\quad \begin{array}{ll}0.59846 \mathrm{ppm} / \mathrm{cm} \\ 239.46362 \mathrm{~Hz} / \mathrm{cm}\end{array}$



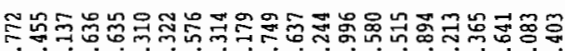
Fi்



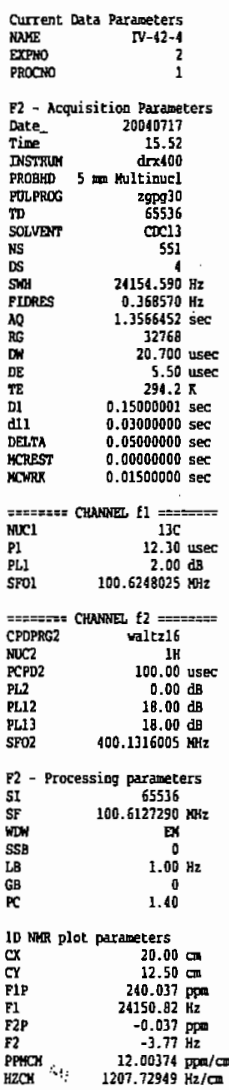





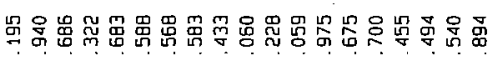

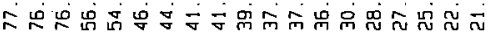
$y+3=1 / 2$

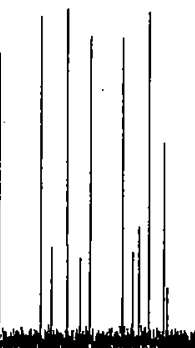

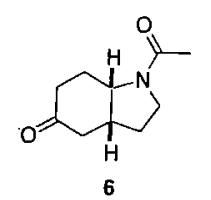

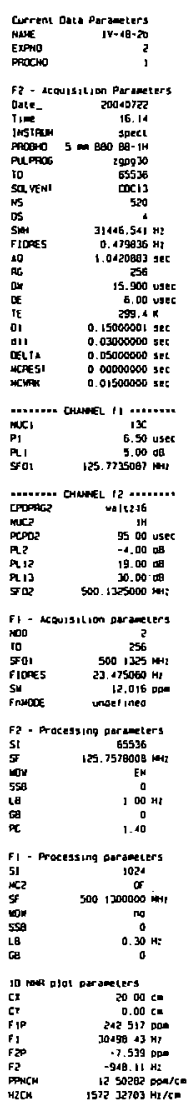




\section{SI-21}
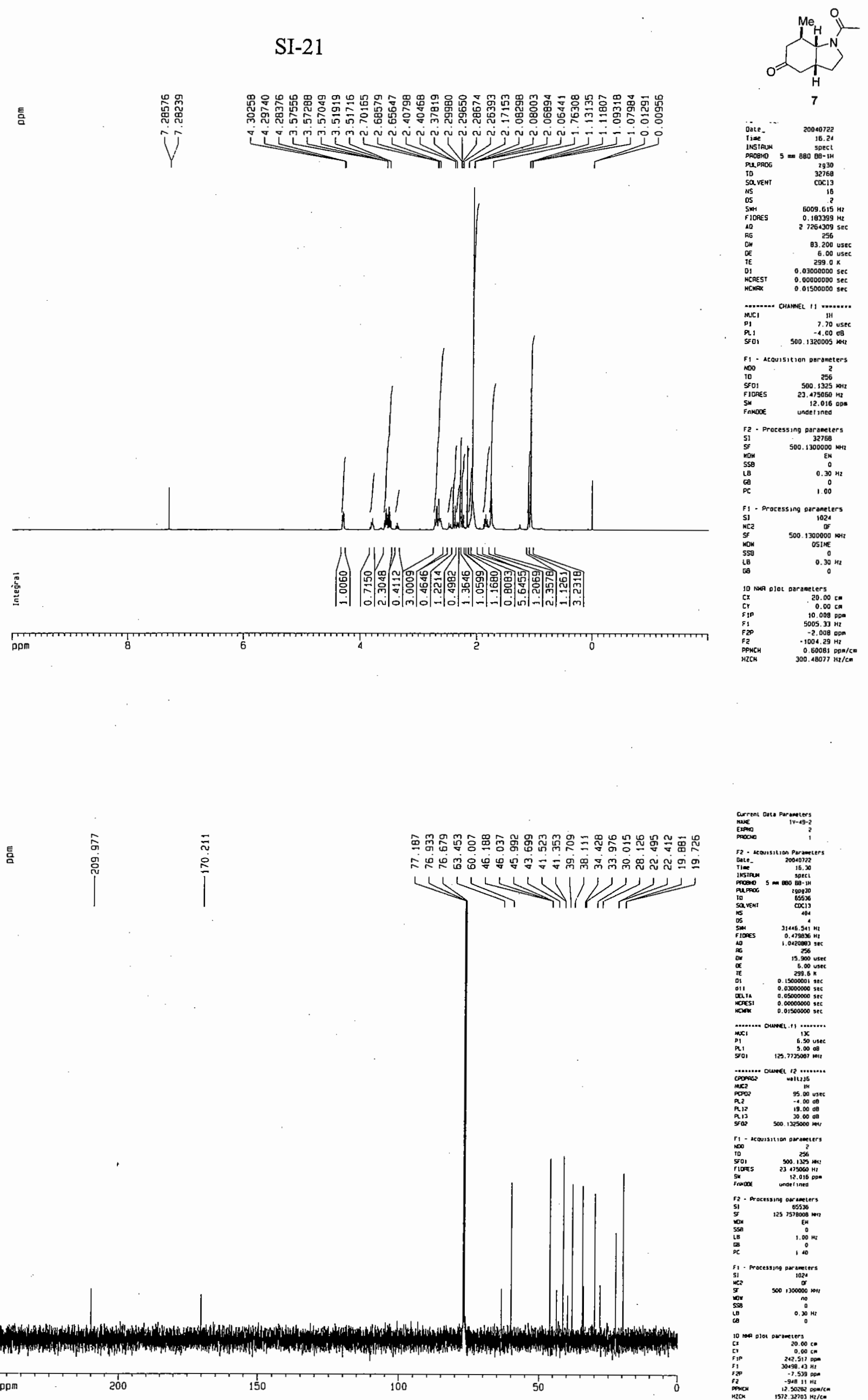



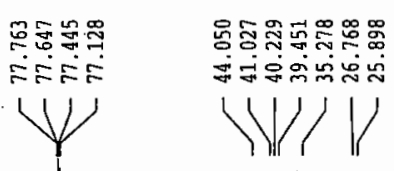

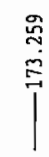

ppm



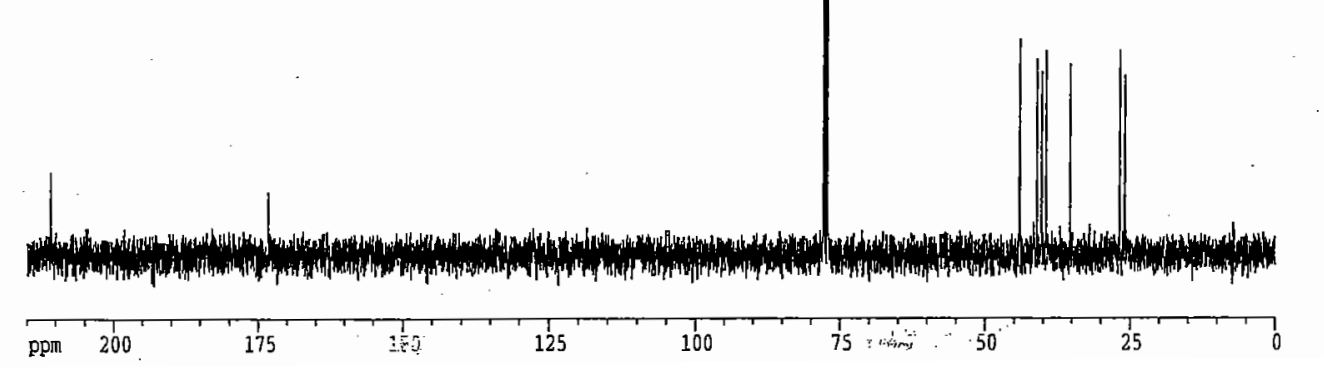

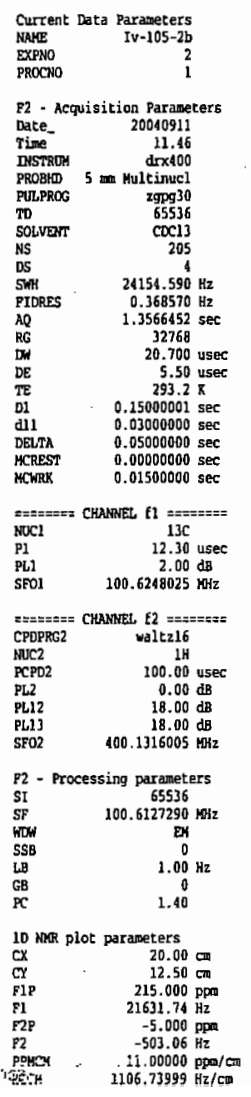




\section{SI-23}
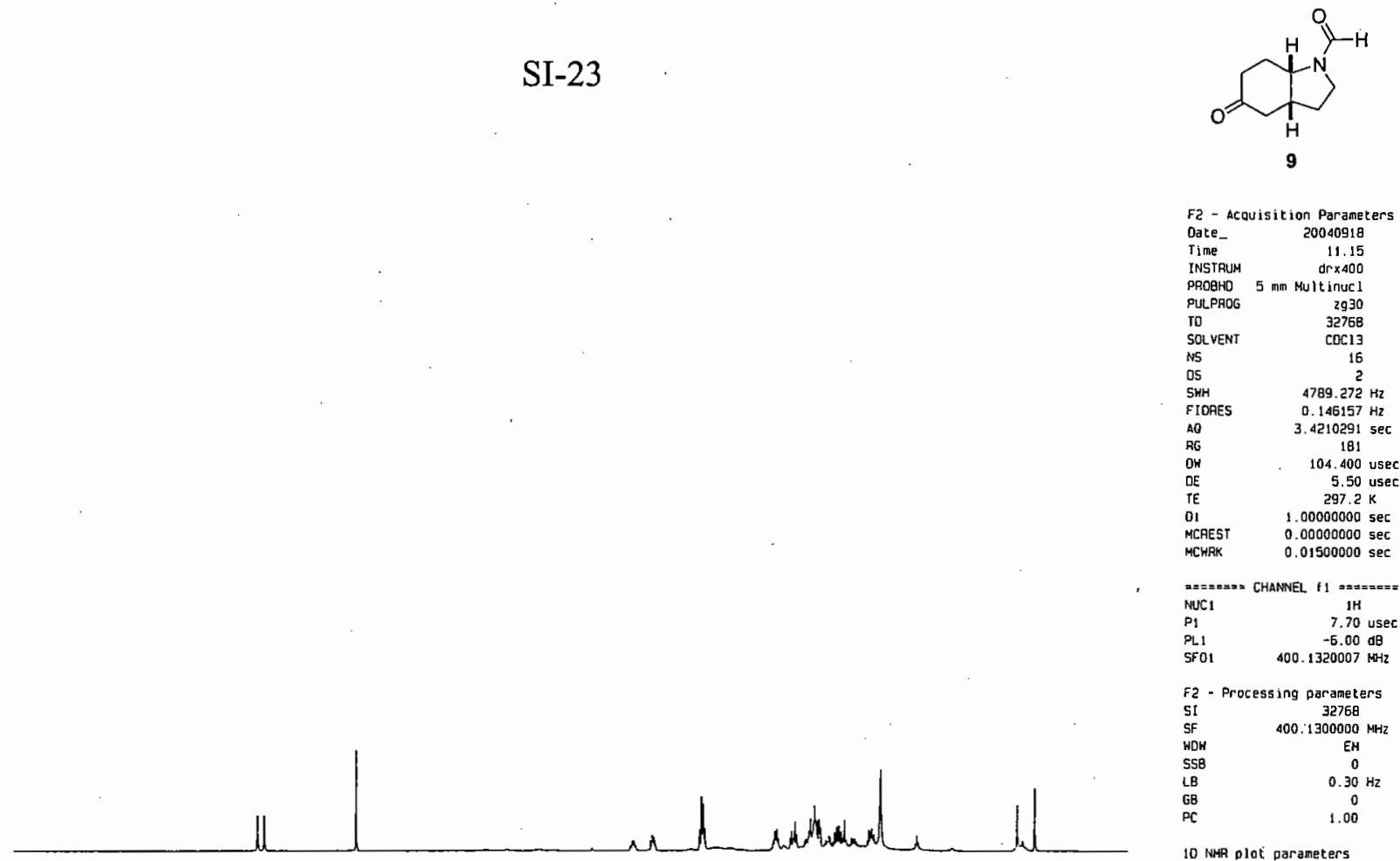

F2 - Processing parameters

SI 32768

SF $\quad 400: 1300000 \mathrm{MH}$

10 Nita olot paraneters

IO Nith plot parameters

$\begin{array}{lr}C X & 20.00 \mathrm{~cm} \\ C Y & 0.00 \mathrm{~cm}\end{array}$

10.985
$\mathrm{dD}$

$\begin{array}{ll}F_{2} \mathrm{P} & -0.985 \mathrm{pp} \\ \mathrm{F}_{2} & -393.99 \mathrm{~Hz}\end{array}$

PPMCM $\quad 0.59846 \mathrm{~Hz}$

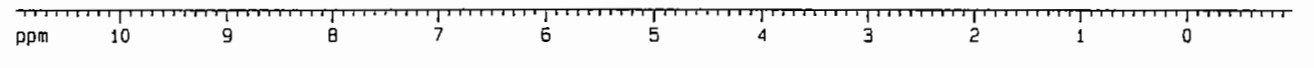

言

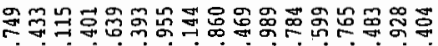

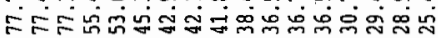

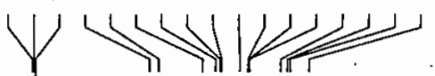

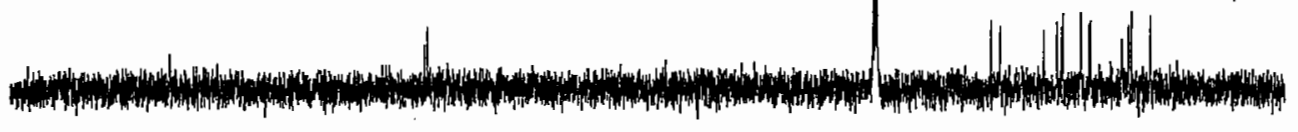

ppm $225 \quad 260, \ldots$ i is
150

125
75
50
25

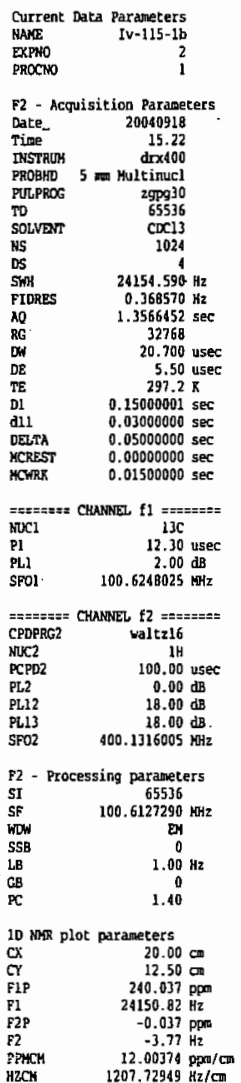



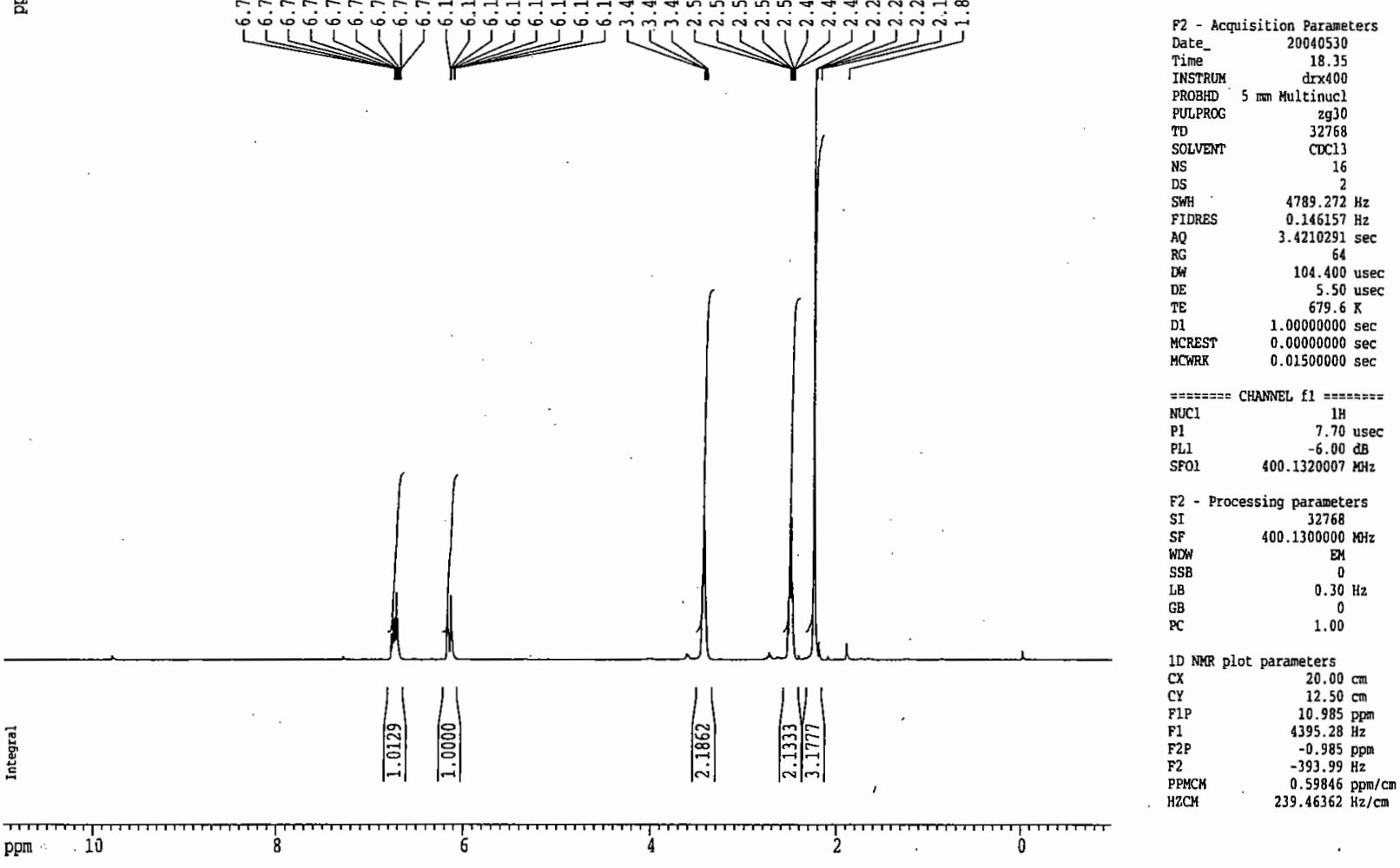

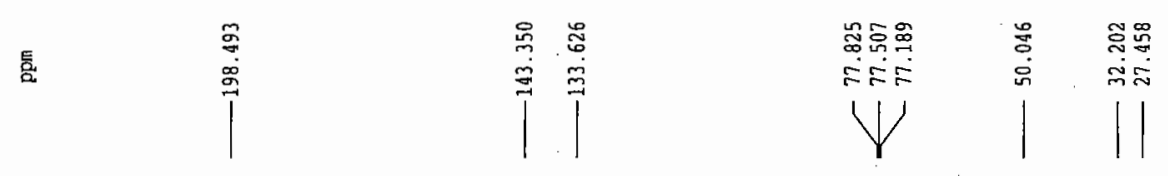

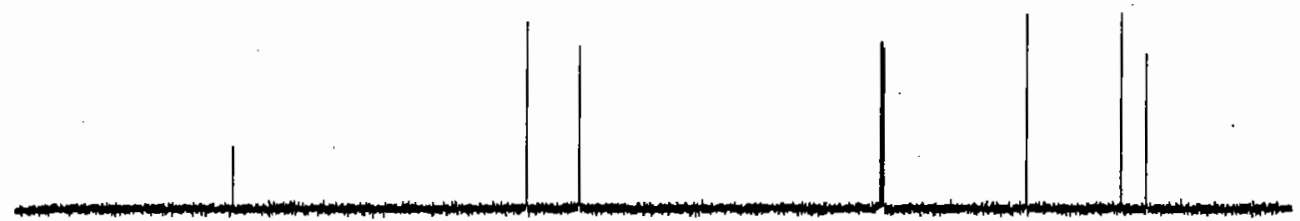

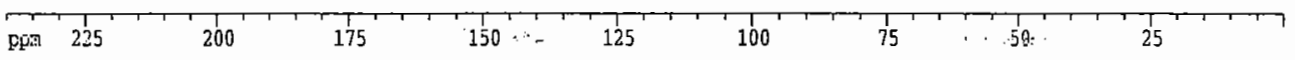

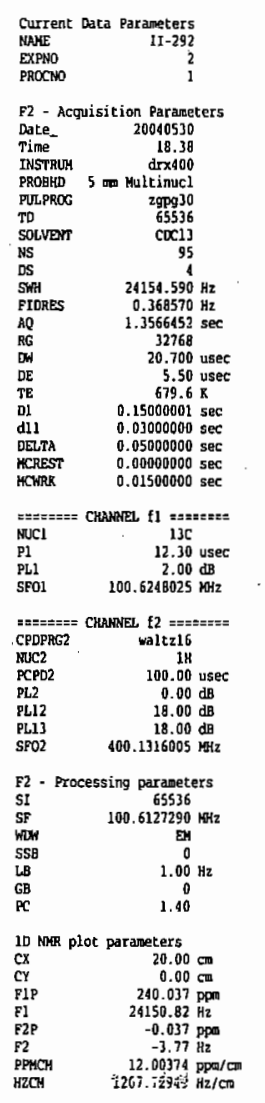




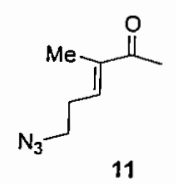

言

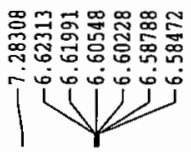

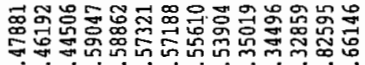

ivivijis

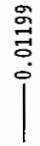

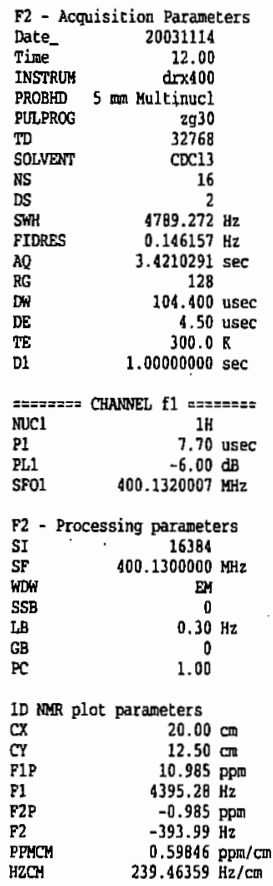

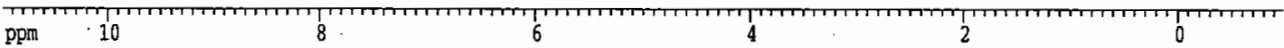
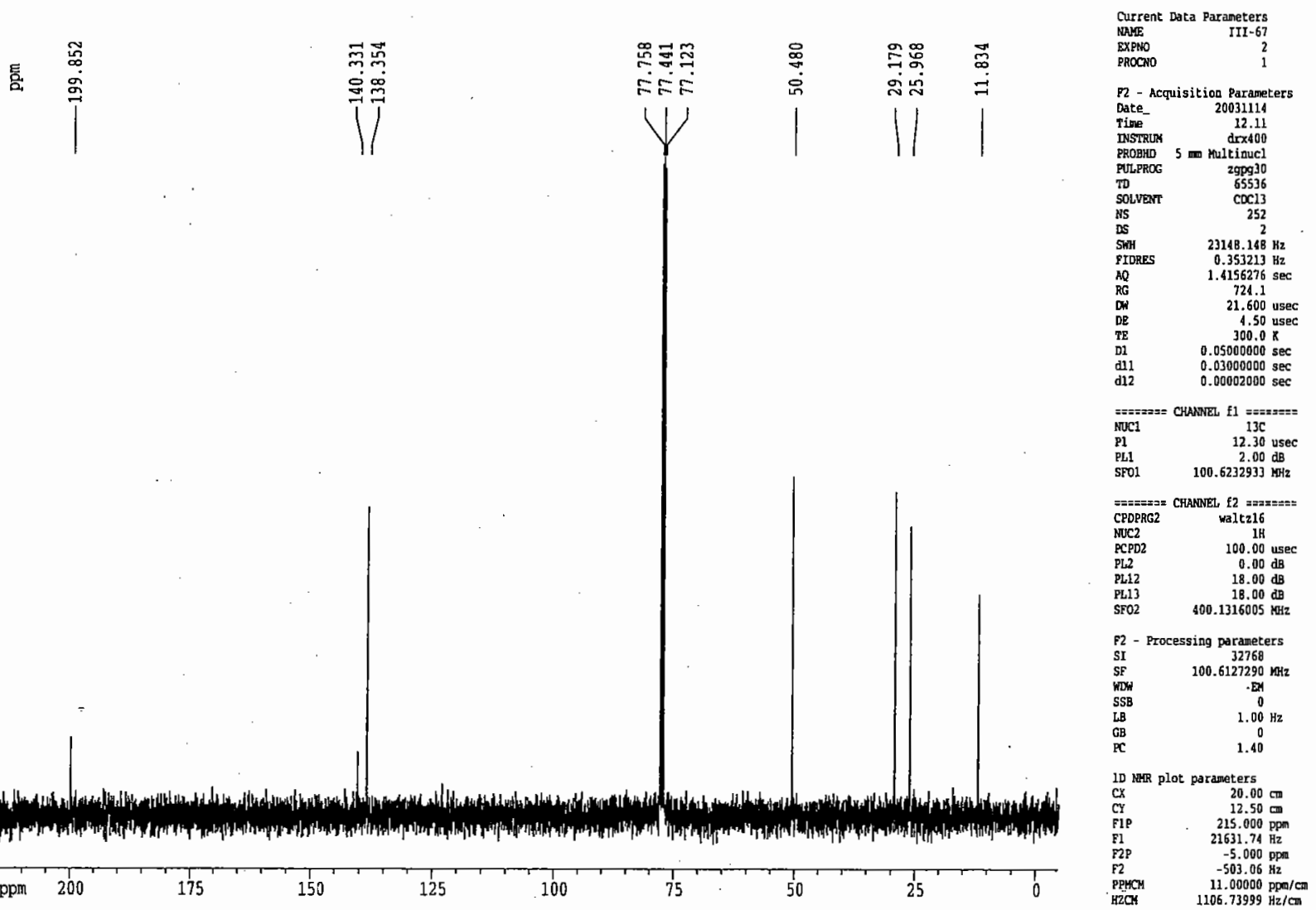


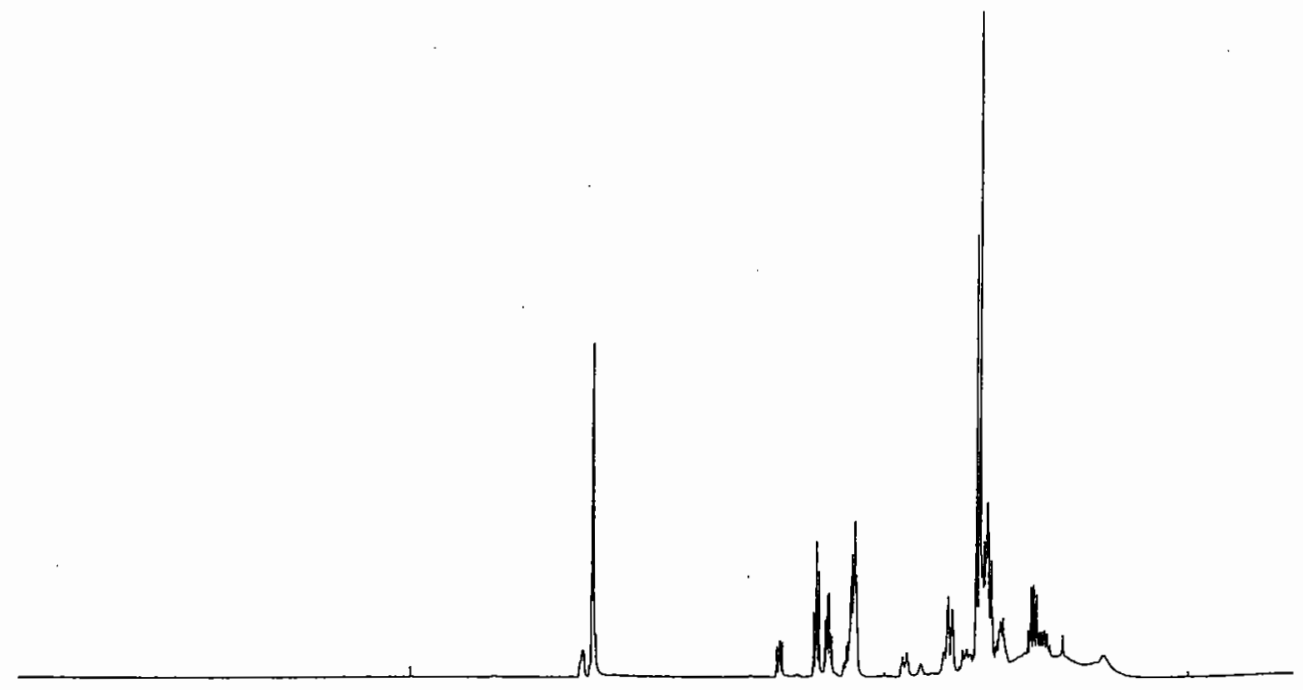

F2 - Acquisjtion Parameters

Date_ Acquss 20030901

Time- 20030901

INSTRUM dr

PAOBHD 5 ma Multinutel

TO
SOLVENT
NS
OS

OS

SNH

AO

AG
OW
DE
TE

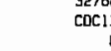

2930
32768
$\operatorname{COC} 13$

$4789.272 \mathrm{~Hz}$

$0.146157 \mathrm{~Hz}$
$3.4210291 \mathrm{sec}$

3. $4210291 \mathrm{sec}$

104. 400 usec

4.50 use

$00000000 \mathrm{sec}$

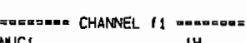

INCS $\quad 7.70$ use

PL1 $\quad-6.00 \mathrm{~dB}$

SFO1 $400.1320097 \mathrm{mHz}$

$\mathrm{FC}$ - Processing parameters

SI 16384

$\begin{array}{lr}\text { SF } & 400.1300000 \\ \text { HDW } & \text { EH }\end{array}$

$\begin{array}{lc}\text { SSB } & 0 \\ \text { L } & 0.30 \mathrm{~Hz} \\ 6 \mathrm{~B} & 0\end{array}$

PC $\quad 1.00$

IO NHA DJot parameters

CX $20.00 \mathrm{~cm}$

CY $\quad-\quad 18.00 \mathrm{~cm}$

$\begin{array}{lr}\text { FIP } & 10.985 \mathrm{pgm} \\ \mathrm{F}_{1} & \mathrm{~d} 395.28 \mathrm{~Hz}\end{array}$

$\begin{array}{ll}\mathrm{F} 2 \mathrm{P} & -0.985 \mathrm{ppm} \\ \mathrm{F2} & -393.99 \mathrm{~Hz}\end{array}$

PPMCM $\quad 0.59846 \mathrm{ppm} / \mathrm{cm}$

$239.46362 \mathrm{~Hz} / \mathrm{cm}$



言



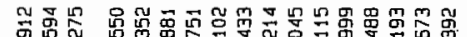

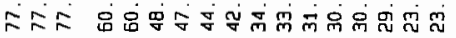

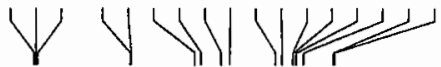

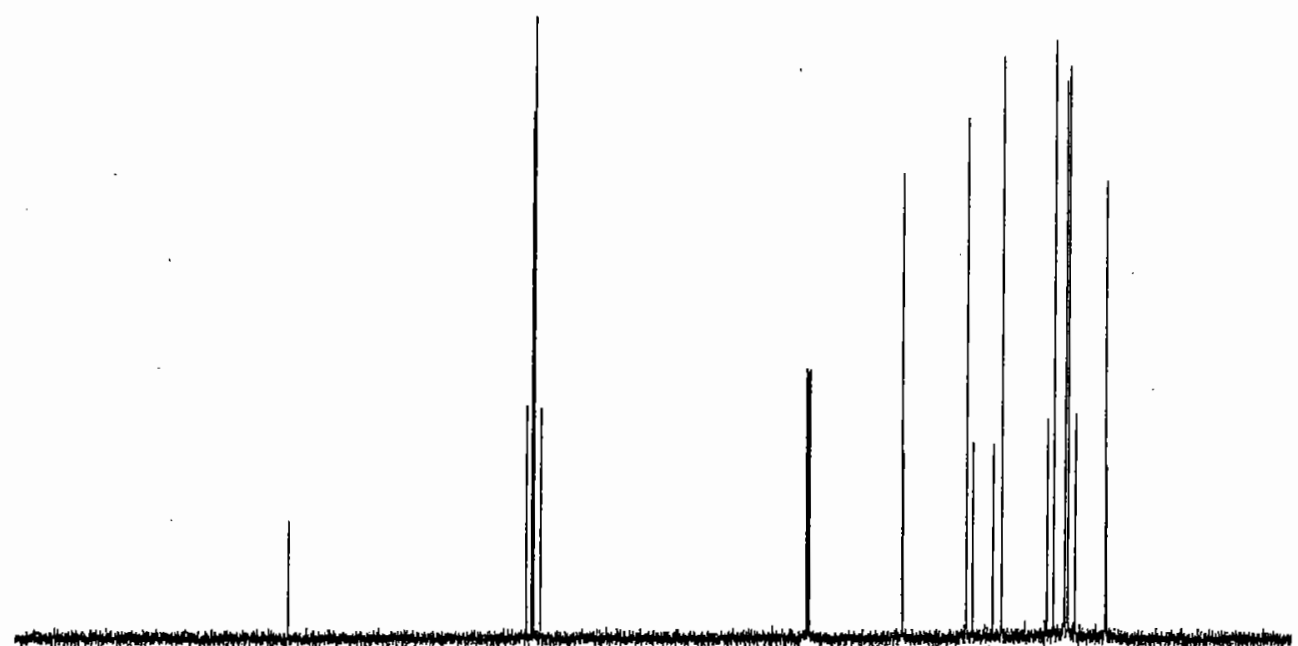

$\rho p m$
200

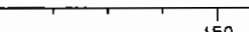

150

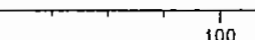

100
50

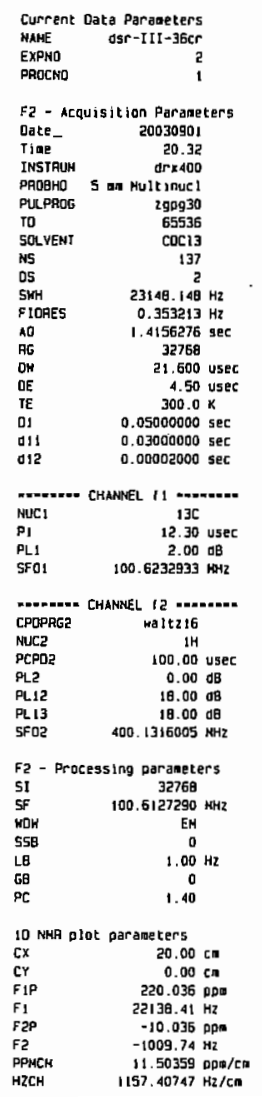



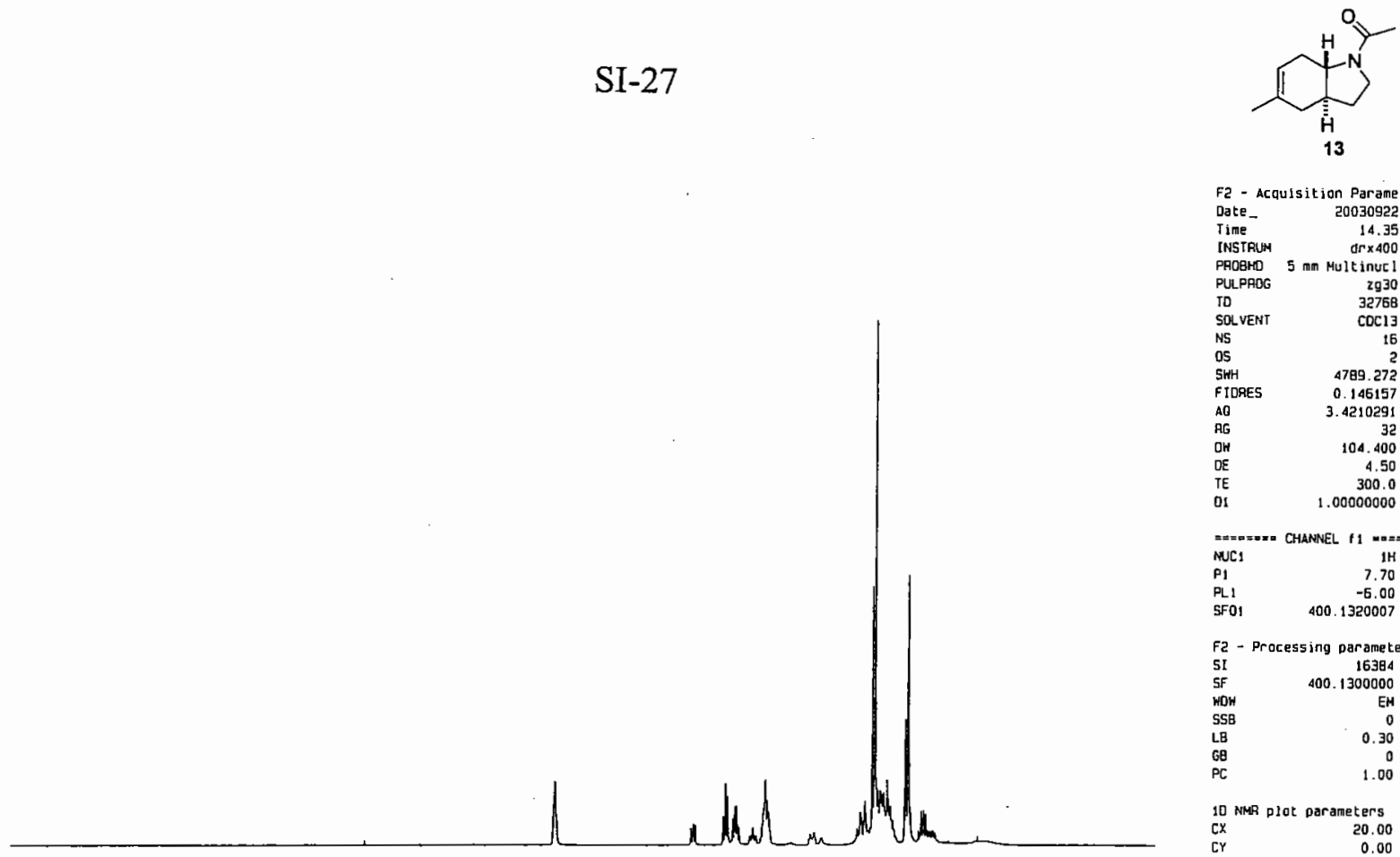

13
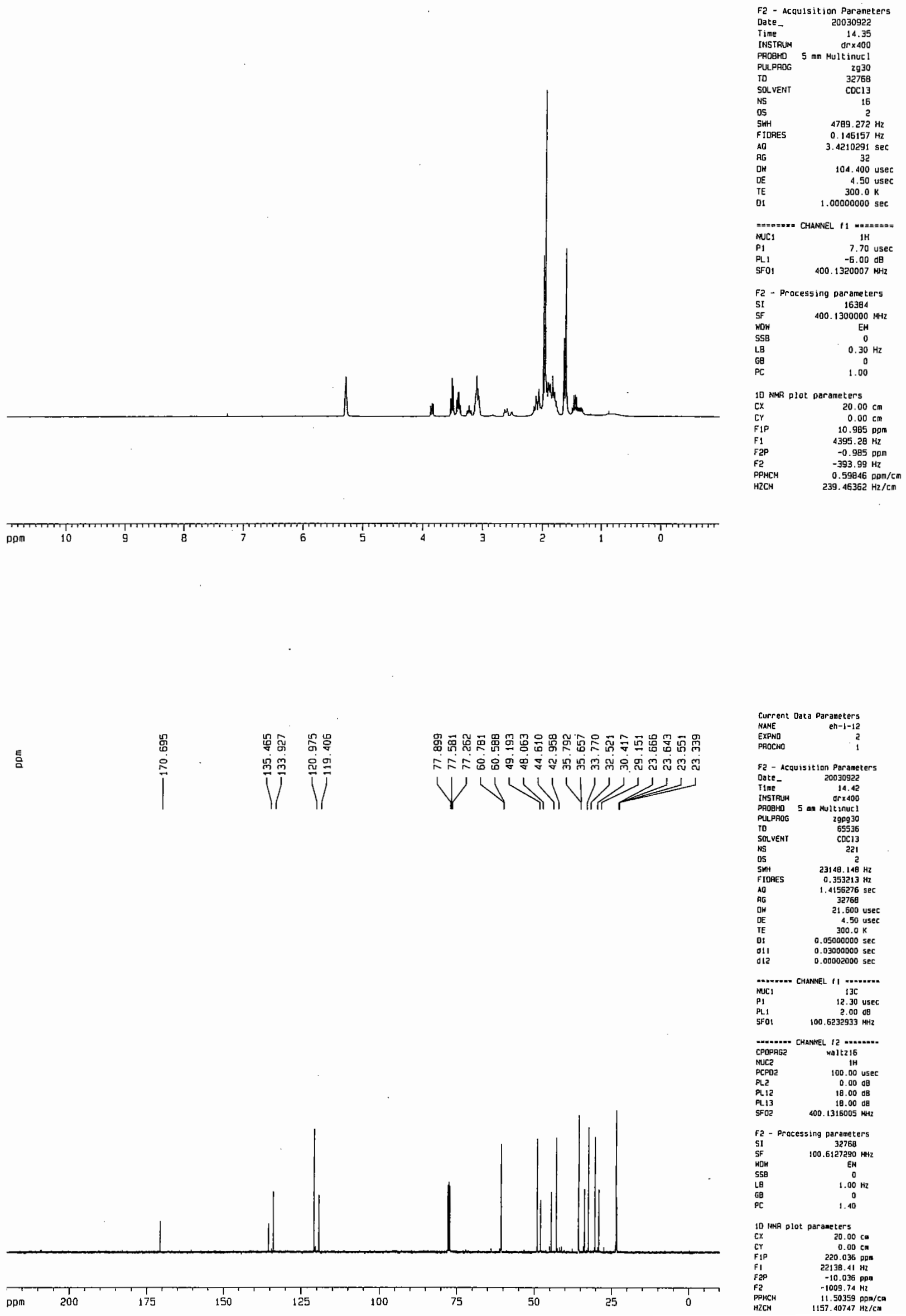


SI-29
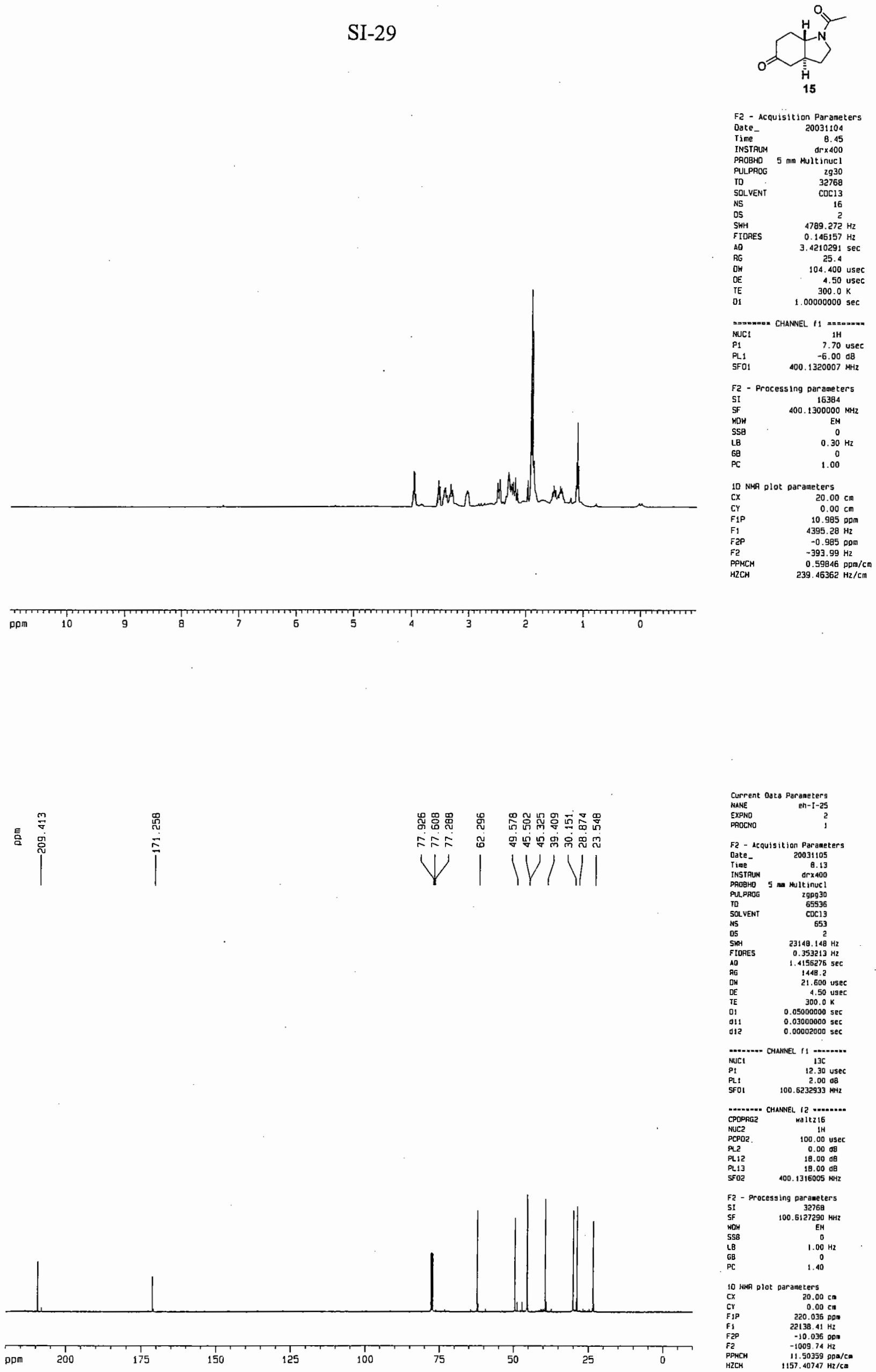


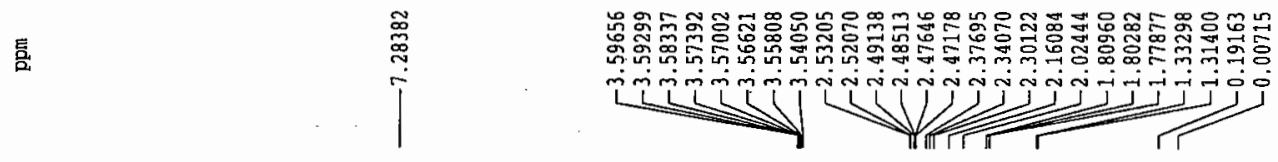

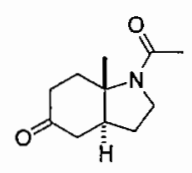

16

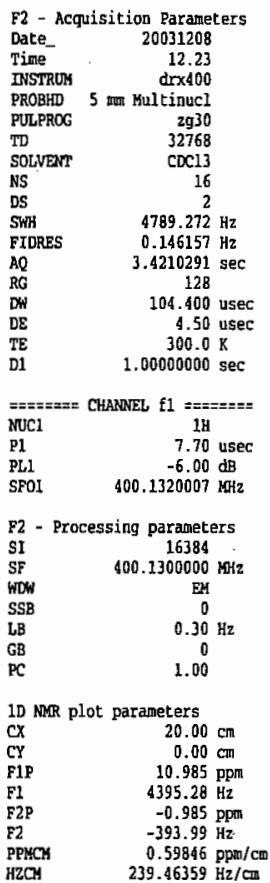

\section{查}

:

荧

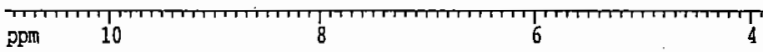

$\mathrm{ppm}_{10}$

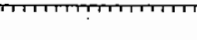

2
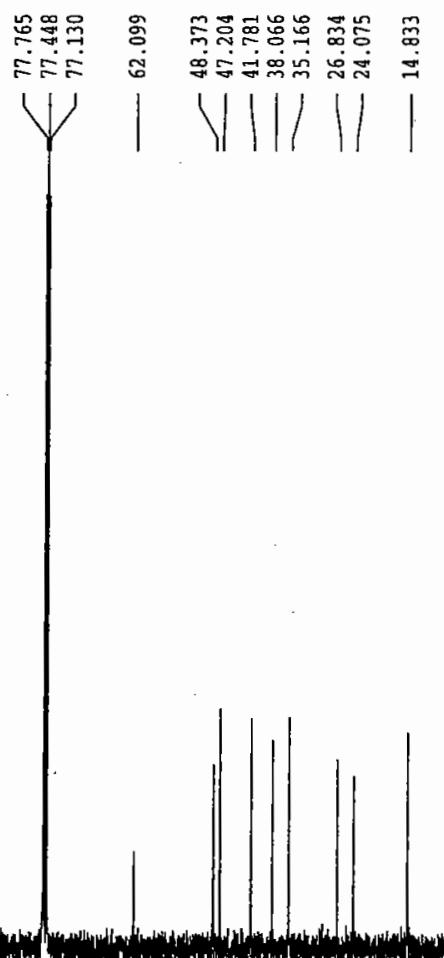

T. The

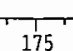

75
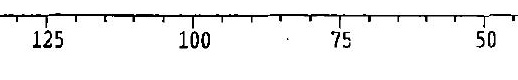

50

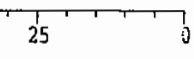

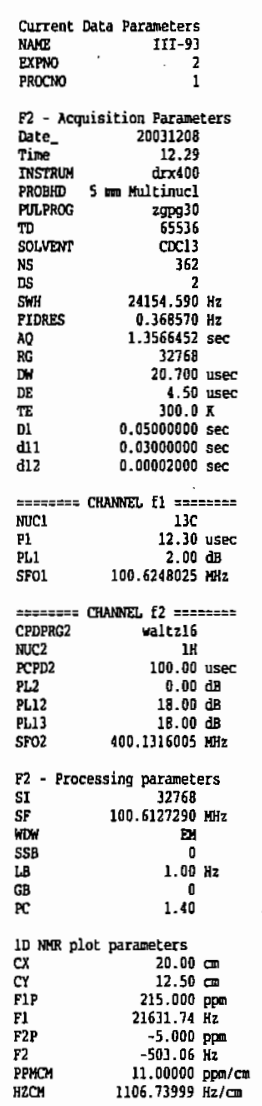




\section{SI-31}
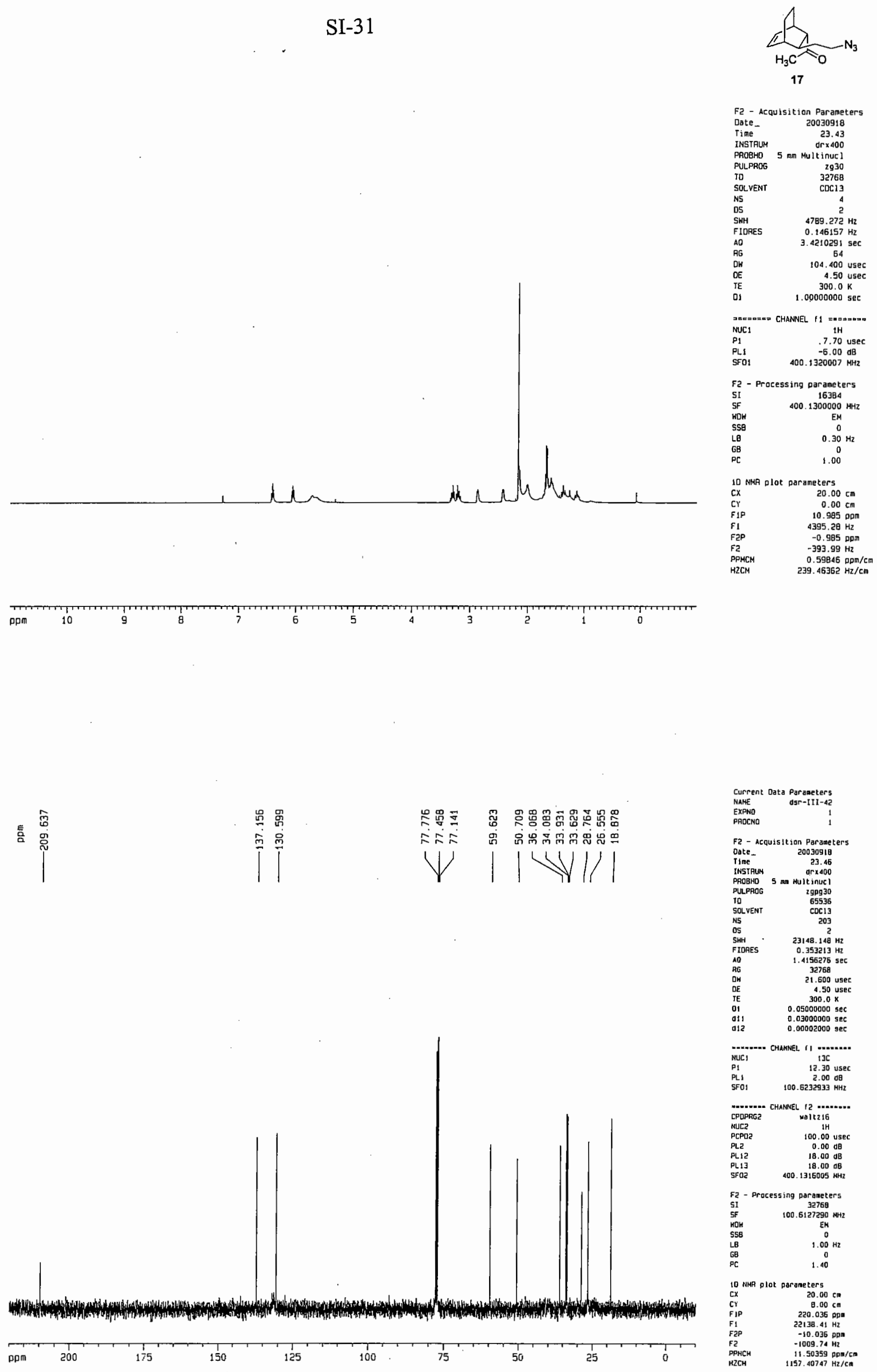


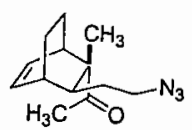

18

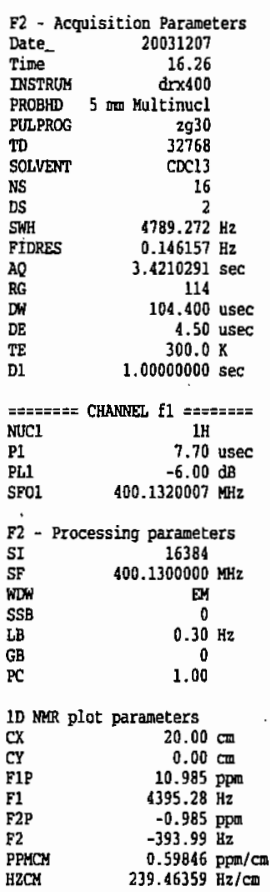


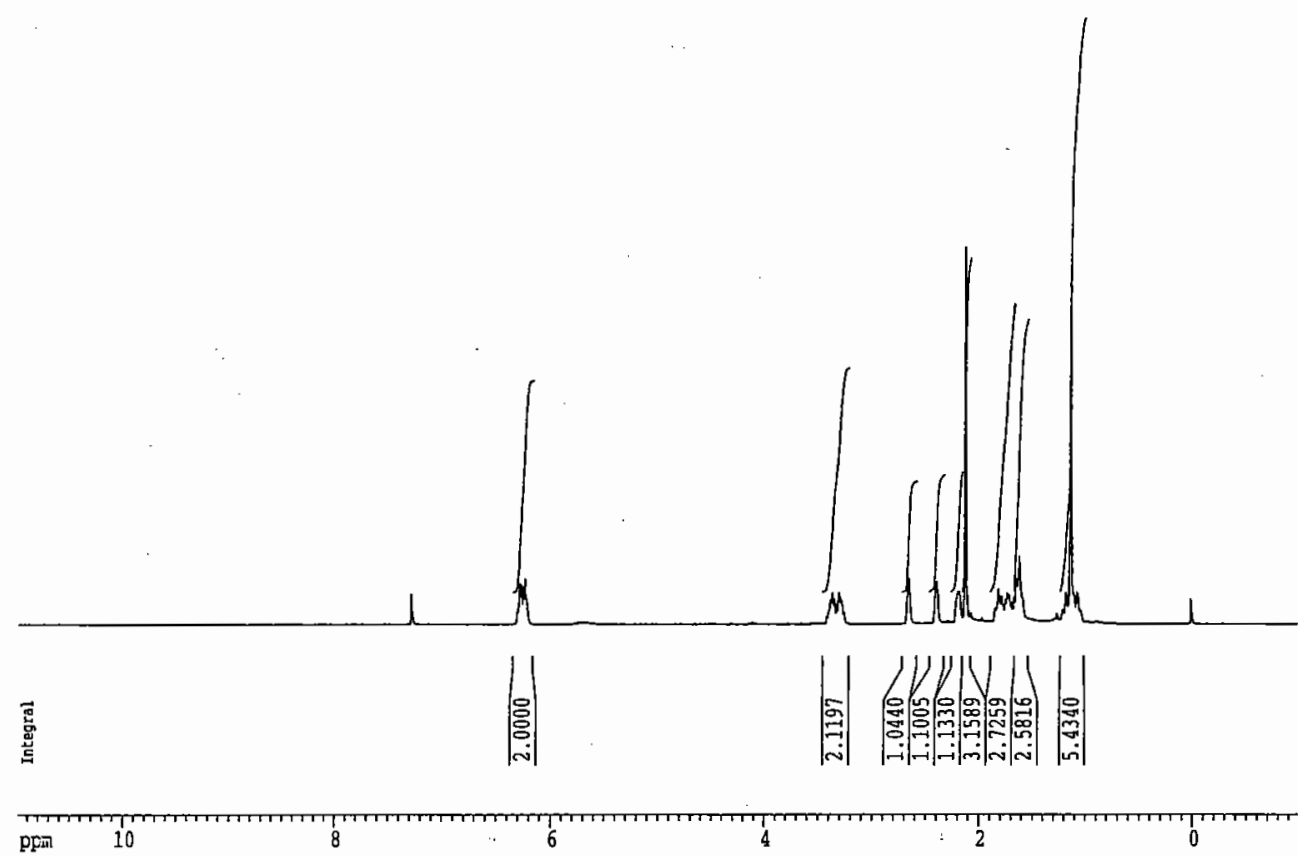

言 $\quad \stackrel{\vec{\sigma}}{\stackrel{7}{7}}$
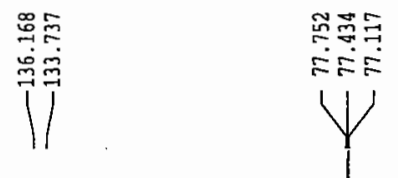

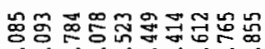


WU1Hit
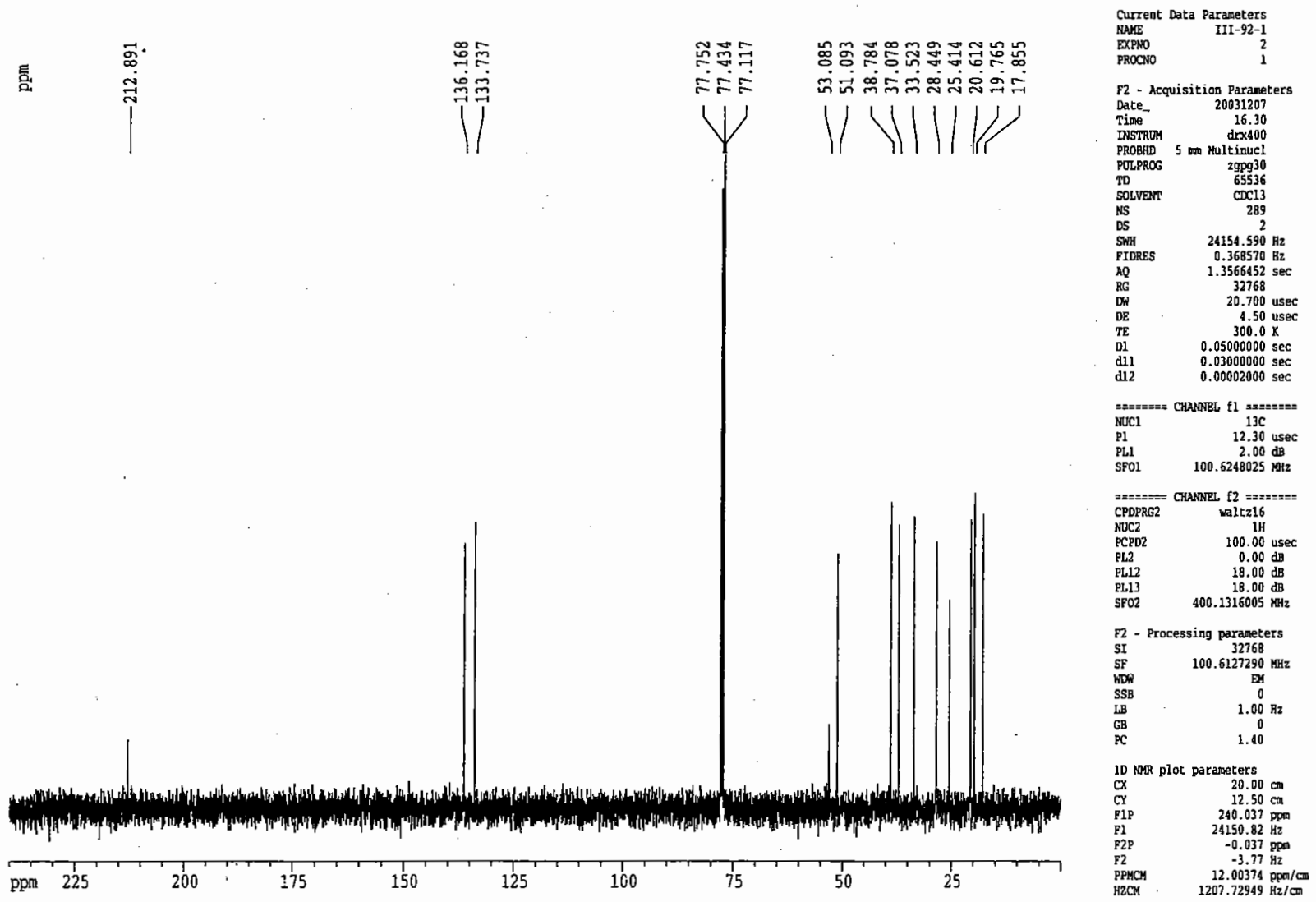

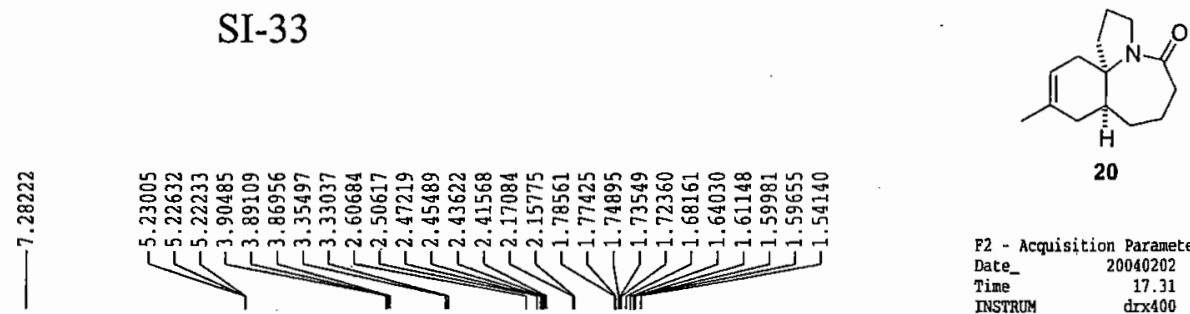

20


$e^{2}$
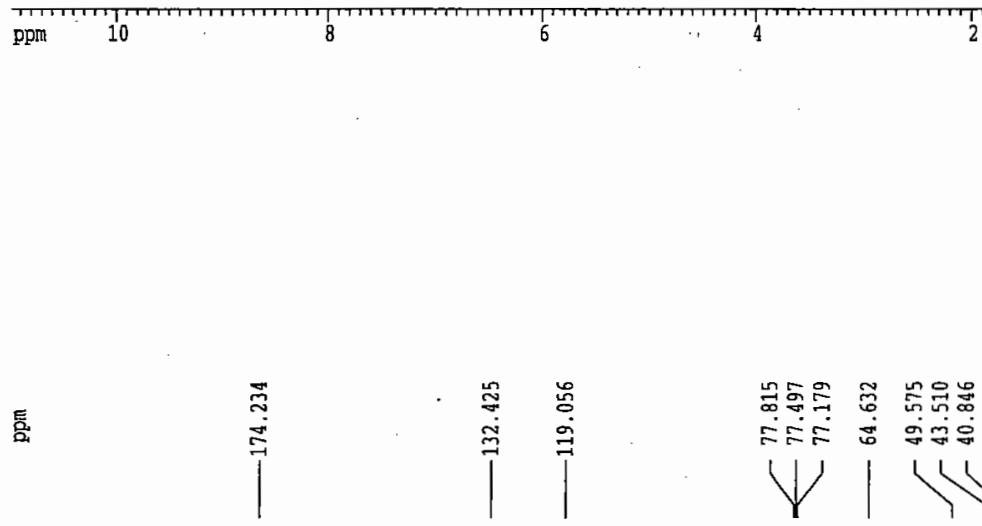

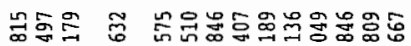

स्टि

$\forall 151 / 1 / 1$

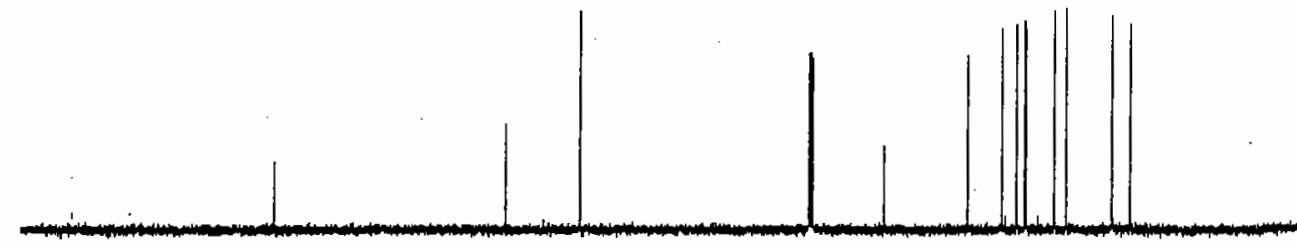



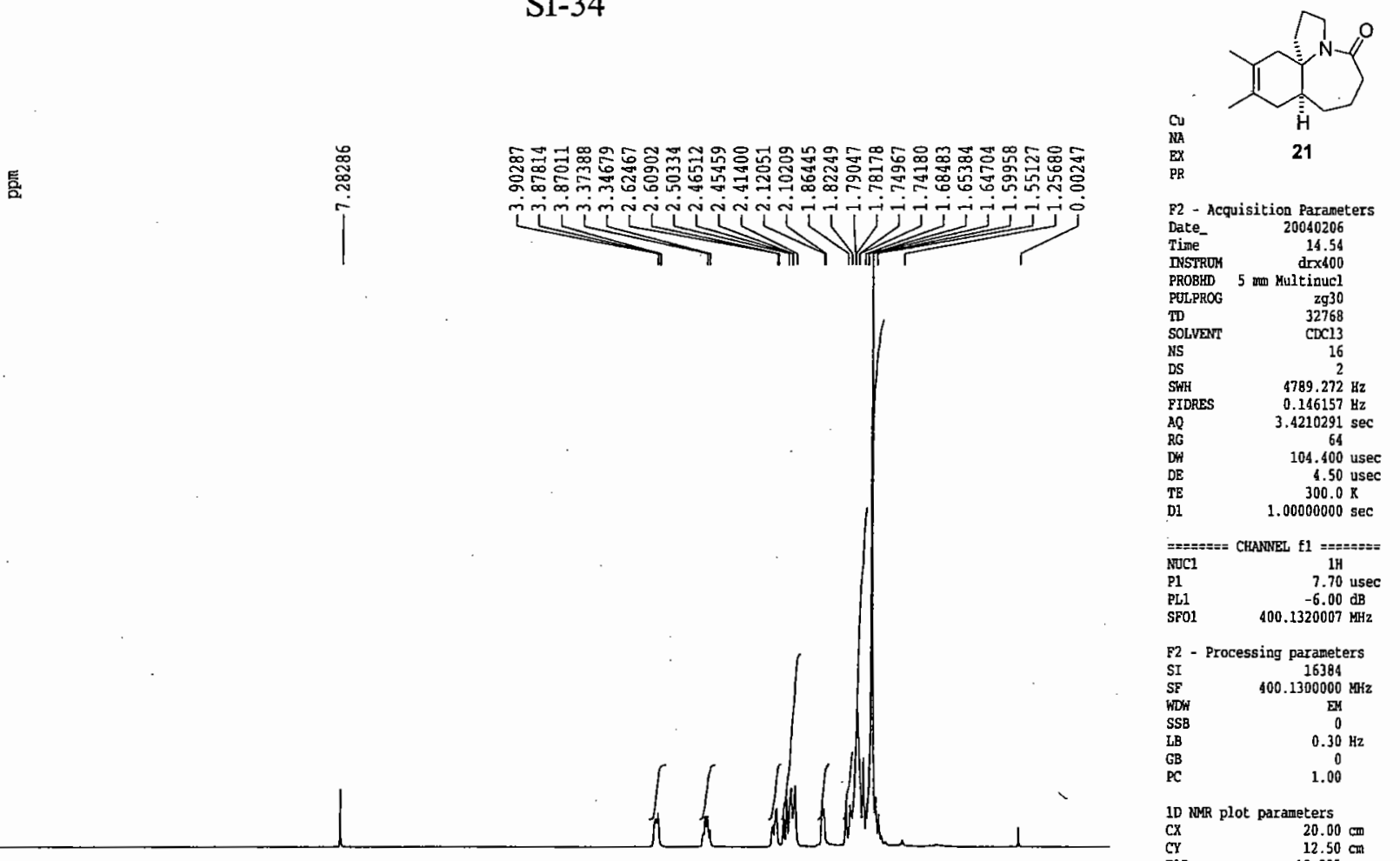

P2 - Acquisition Paraneters

Date_ 20040206

$\begin{array}{lr}\text { Time } & 14.54 \\ \text { DNSTROM } & \text { drx400 } \\ \text { PROBHD } & 5 \text { wn Multinucl }\end{array}$

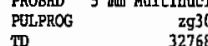

Solva

$\begin{array}{lc}\text { NS } & 16 \\ \text { DS } & 2 \\ \text { SWH } & 4789.272 \mathrm{~Hz}\end{array}$

FIDRES $\quad 0.146157 \mathrm{~Hz}$

$\begin{array}{ll}\text { AQ } & 3.4210291 \mathrm{sec} \\ \text { RG } & 64\end{array}$

$\begin{array}{rr}\mathrm{D} & 64 \\ \mathrm{DW} & 104.400 \mathrm{usec} \\ \mathrm{DE} & 4.50 \text { usec }\end{array}$

$\begin{array}{rr}\mathrm{DE} & 4.50 \text { use } \\ \mathrm{TE} & 3000 \mathrm{~K}\end{array}$

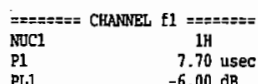

SF01 $\quad 400.1320027 \mathrm{kHz}$

F2 - Processing parameters

$\begin{array}{lr}\text { SI } & 16384 \\ \text { SF } & 400.1300000 \mathrm{HHF}\end{array}$

WDW $\quad$ EM

$\begin{array}{lc}\text { SSB } & 0 \\ \mathrm{LB} & 0.30 \mathrm{~Hz} \\ \mathrm{~GB} & 0 \\ \mathrm{PC} & 1.00\end{array}$

1.00

ID NMR plot paraneters $\begin{array}{ll}\mathrm{CX} & 28.00 \mathrm{~cm} \\ \mathrm{CY} & 12.50 \mathrm{~cm}\end{array}$

FI $\quad 10.985 \mathrm{ppp}$

$\begin{array}{ll}\text { F2P } & -0.985 \\ \text { F2 } & -393.99 \mathrm{ppn}\end{array}$

$\begin{array}{lr}\text { PPRCM } & 0.59846 \mathrm{ppm} / \mathrm{cm} \\ \text { HZCM } & 239.46359 \mathrm{~Hz} / \mathrm{cm}\end{array}$

营
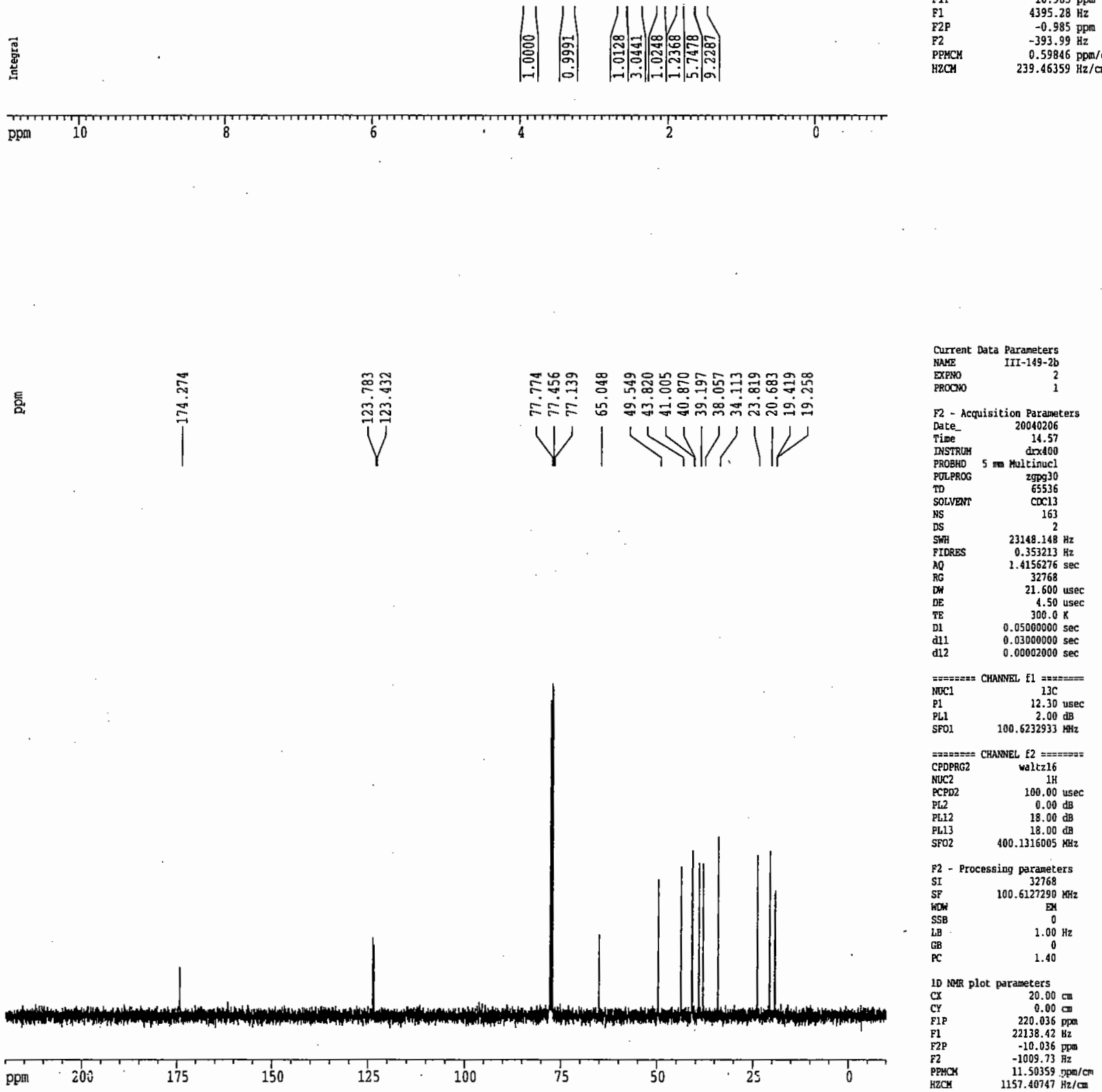



동 में

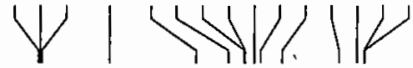





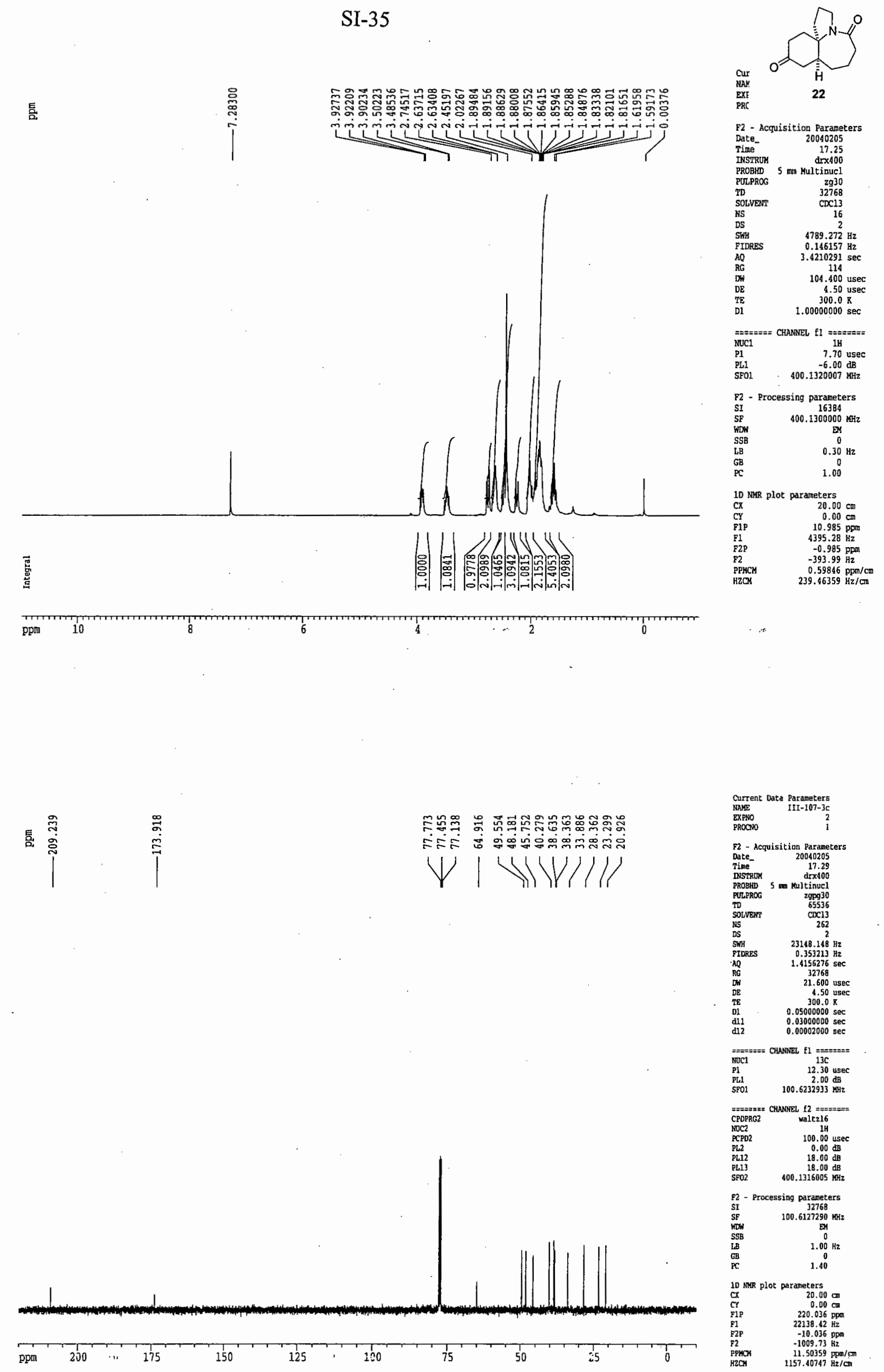